\title{
SDSS-IV MaNGA: Evidence of the importance of AGN feedback in low-mass galaxies
}

\author{
Samantha J. Penny, ${ }^{\star}$ Karen L. Masters, ${ }^{1}$ Rebecca Smethurst, ${ }^{2}$ Robert C. Nichol, ${ }^{1}$ \\ Coleman M. Krawczyk, ${ }^{1}$ Dmitry Bizyaev, ${ }^{3,4}$ Sandro B. Rembold, ${ }^{5}$ \\ Rogemar A. Riffel, ${ }^{5,6}$ Gabriele da Silva Ilha, ${ }^{5}$ Brett H. Andrews, ${ }^{7}$ Kevin Bundy, ${ }^{8}$ \\ Niv Drory, ${ }^{9}$ Daniel Oravetz, ${ }^{3}$ Kaike Pan ${ }^{3}$ \\ ${ }^{1}$ Institute of Cosmology and Gravitation, University of Portsmouth, Burnaby Road, Portsmouth PO1 3FX, UK \\ ${ }^{2}$ School of Physics and Astronomy, University of Nottingham, University Park, Nottingham, NG7 2RD, UK \\ ${ }^{3}$ Apache Point Observatory and New Mexico State University, P.O. Box 59, Sunspot, NM, 88349-0059, USA \\ ${ }^{4}$ Sternberg Astronomical Institute, Moscow State University, Moscow, Russia \\ ${ }^{5}$ Departamento de Física, CCNE, Universidade Federal de Santa Maria, 97105-900, Santa Maria, RS, Brazil \\ ${ }^{6}$ Laboratório Interinstitucional de e-Astronomia, 77 Rua General José Cristino, Rio de Janeiro, 20921-400, Brasil \\ ${ }^{7}$ PITT PACC, Department of Physics and Astronomy, University of Pittsburgh, Pittsburgh, PA 15260, USA \\ ${ }^{8}$ University of California, Santa Cruz, 1156 High St. Santa Cruz, CA 95064, USA \\ ${ }^{9}$ McDonald Observatory, The University of Texas at Austin, 1 University Station, Austin, TX 78712, USA
}

Accepted XXX. Received YYY; in original form ZZZ

\begin{abstract}
We present new evidence for AGN feedback in a subset of 69 quenched low-mass galaxies $\left(M_{\star} \lesssim 5 \times 10^{9} \mathrm{M}_{\odot}, M_{\mathrm{r}}>-19\right)$ selected from the first two years of the SDSSIV MaNGA survey. The majority (85 per cent) of these quenched galaxies appear to reside in a group environment. We find 6 galaxies in our sample that appear to have an active AGN that is preventing on-going star-formation; this is the first time such a feedback mechanism has been observed in this mass range. Interestingly, five of these six galaxies have an ionised gas component that is kinematically offset from their stellar component, suggesting the gas is either recently accreted or outflowing. We hypothesise these six galaxies are low-mass equivalents to the "red geysers" observed in more massive galaxies. Of the other 62 galaxies in the sample, we find 8 do appear for have some low-level, residual star formation, or emission from hot, evolved stars. The remaining galaxies in our sample have no detectable ionised gas emission throughout their structures, consistent with them being quenched. This work shows the potential for understanding the detailed physical properties of dwarf galaxies through spatially resolved spectroscopy.
\end{abstract}

Key words: galaxies: evolution - galaxies: active - galaxies: kinematics and dynamics - galaxies: dwarf

\section{INTRODUCTION}

The role of nature versus nurture, and their respective contributions to galaxy evolution, are unclear. We know that a large fraction of high-mass galaxies in all environments have ceased star formation (e.g. Di Matteo et al. 2005; Ilbert et al. 2010; Peng et al. 2010), and those massive galaxies in isolation must therefore be able to shut-off and prevent further star formation via processes internal to the galaxies themselves. It is also well known that low-mass and

^ E-mail: samantha.penny@port.ac.uk (SJP) satellite galaxies in medium to high density environments have ceased star formation (e.g. Peng et al. 2010; Geha et al. 2012; Smith et al. 2012; Wetzel et al. 2013). One of the remaining uncertainties is the role that AGN and other intrinsic processes play in the evolution of low mass galaxies with stellar masses $M_{\star}<5 \times 10^{9} \mathrm{M}_{\odot}$.

Geha et al. (2012) showed that the majority of quenched dwarf galaxies with stellar masses $M_{\star}<10^{9} \mathrm{M}_{\odot}$ are found in regions of high local galaxy density, such that > 99 per cent of quenched dwarfs in the Sloan Digital Sky Survey (SDSS, York et al. 2000) are found within a projected distance of $1.5 \mathrm{Mpc}$ and $\pm 1000 \mathrm{~km} \mathrm{~s}^{-1}$ from a galaxy with $M_{\mathrm{K}}<-23$. Very few quenched dwarfs are found in iso- 
lation (though see Janz et al. 2017), so environmental processes are likely required for their initial gas removal. However, dwarf galaxies with clear AGN signatures at optical and mid-IR wavelengths are also found (Reines et al. 2013; Moran et al. 2014; Sartori et al. 2015). It is therefore unclear whether low-mass galaxies can be efficiently quenched or even influenced by AGN activity, or if other processes are required to shut-off their star formation. Do these AGN act to maintain quiescence in low mass galaxies, in a process similar to the red-geysers identified in Cheung et al. (2016)?

The shape of the galaxy luminosity/mass function is often explained using feedback mechanisms, such that above a characteristic mass/luminosity turnover at $M_{\star} \approx 10^{10} \mathrm{M}_{\odot}$, AGN feedback is dominant in driving galaxy evolution and regulating star formation, while below this mass, supernova feedback or winds from high-mass stars dominate (e.g. Bower et al. 2006; Schaye et al. 2015). As a result, in many galaxy evolution models, AGN feedback is only invoked for galaxies with $M_{\star}>10^{10} \mathrm{M}_{\odot}$. However, Smethurst et al. (2016) showed that the effects of AGN feedback have the biggest impact on the stellar populations of low-to-intermediate mass galaxies with $M_{\star}<10^{10.25} \mathrm{M}_{\odot}$ in a sample of 1,244 BPT selected AGN from SDSS. Recently, Dashyan et al. (2017) have proposed AGN feedback as a feedback and quenching mechanism for dwarf galaxies, which is able to drive gas out of dwarf-sized haloes more efficiently than supernova feedback. Further complicating our picture of low-mass galaxy evolution is that a fraction of bright dwarf galaxies with $M_{\star} \sim 10^{9} \mathrm{M}_{\odot}$ are the remnants of morphologically transformed disk galaxies, and as such, many retain evidence of this (e.g. Lisker et al. 2006; Penny et al. 2014; Toloba et al. 2015; Penny et al. 2016). This structure includes faded spiral features and embedded disks, disc-like kinematics, and may include central supermassive black holes.

AGN are often going to be missed in wide-field searches for quenched galaxies. If a galaxy exhibits red optical colours consistent with quiescence, yet hosts strong central emission, it might be removed from a quenched galaxy sample if just the strength or equivalent width of the $\mathrm{H} \alpha$ line is used as a diagnostic of active star formation. In contrast, but also problematic in understanding if AGN influence the evolution of bright dwarf ellipticals, is that low mass galaxies in clusters (the most commonly studied dEs) are often gas poor objects. The central black holes in such galaxies will therefore be undetectable through emission line diagnostics, and we cannot learn anything about the role of their AGN phase in their evolution.

Indeed, evidence for AGN has been found in the centre of ultra compact dwarfs (Seth et al. 2014; Ahn et al. 2017), thought to be the nuclear remnants of tidally stripped disk galaxies (e.g. Drinkwater et al. 2003; Pfeffer \& Baumgardt 2013). The existence of AGN has also been identified in several dwarf-mass galaxies, through optical emission line diagnostics, X-ray observations, mid-IR diagnostics, and radio continuum surveys. For example, Lemons et al. (2015) cross-matched 44,000 dwarf galaxies in the NASA Sloan Atlas, and found $\sim 10$ galaxies with nuclear X-ray sources. Sartori et al. (2015) find that just 0.7 per cent of galaxies with $M_{\star}<5 \times 10^{9} \mathrm{M}_{\odot}$ have clear signs of hosting an AGN using the BPT diagram, He II $\lambda 4686$ line, and mid-IR colours as AGN diagnostics. More recently, Nucita et al. (2017) have found 51 low-mass galaxies with $M_{\star}<10^{10} \mathrm{M}_{\odot}$ that host Xray sources, of which 37 per cent are centrally located within their host galaxy, a number of which also have radio counterparts.

Low mass quenched galaxies are ubiquitous in groups and clusters, and understanding the role that AGN feedback plays in their evolution is therefore crucial for their modelling. To detect AGN in low mass galaxies, the group environment may prove more fruitful than searches in nearby clusters such as Virgo and Coma. The weaker tidal potential of galaxy groups versus clusters, along with the lower-density intra-group medium means bright dEs in galaxy groups will likely retain more of their gas component than their cluster counterparts. Thus a wide-field Integral Field Unit (IFU) study that includes galaxies with $M_{\star}<5 \times 10^{9} \mathrm{M}_{\odot}$ is needed to fully understand the role that AGN play in the evolution of low-mass galaxies.

In this work, we aim to identify candidate dwarf AGN hosted by low-mass galaxies in the SDSS-IV MaNGA survey, which includes low-mass galaxies in all environments with $M_{\star}<5 \times 10^{9} \mathrm{M}_{\odot}$. In particular, we aim to identify red geyser candidates (Cheung et al. 2016) in the low-mass galaxy sample, which typically have kinematically offset ionised gas, either the result of accretion or outflows of material generated by AGN-driven winds. Spatial data is required to map these stellar and ionised gas velocity components, as well as identify the bi-symmetric emission line features seen in red geysers which would not be identified in single-fibre or long-slit spectroscopic surveys.

We present an overview of the MaNGA observations used for this work in Section 2, with the sample selection described in Section 2.2. We examine the ionised gas emission line mechanism in Section 3, with spaxel-by-spaxel emission line diagnostics presented in Section 3.1, and mid-IR colours for the quenched, low-mass galaxies examined in Section 3.3. We discuss our results in Section 4, and conclude in Section 5. Throughout this paper, we assume a $\Lambda$ CDM cosmology, with $H_{0}=70 \mathrm{~km} \mathrm{~s}^{-1}, \Omega_{M}=0.3$, and $\Omega_{\Lambda}=0.7$. All magnitudes are presented in the $\mathrm{AB}$ magnitude system, unless otherwise stated.

\section{OBSERVATIONS AND SAMPLE SELECTION}

\subsection{The MaNGA Survey}

The data for this work is drawn from the Sloan Digital Sky Survey IV (SDSS-IV, Blanton et al. 2017) Mapping Nearby Galaxies at APO (MaNGA, Bundy et al. 2015) survey. Searching for the processes responsible for quenching requires high quality, spatially resolved spectroscopic data, in order to trace key spectral features that will reveal the quenching mechanisms active in a given galaxy. MaNGA is a multi-object IFU survey using the Baryon Oscillation Spectroscopic Survey (BOSS) spectrograph (Smee et al. 2013) on the $2.5 \mathrm{~m}$ Sloan Foundation Telescope (Gunn et al. 2006). MaNGA will target 10,000 galaxies with $M_{\star} \gtrsim 10^{9} \mathrm{M}_{\odot}$ by the completion of the survey in 2020, which began taking data on 2014 July 1. The MaNGA targets are selected independent of colour, morphology and environment, ensuring we draw targets from a range of local galaxy density, crucial in a study of the processes responsible for galaxy quenching. 
MaNGA has a spectral resolution $R \sim 2000$, which corresponds to an instrumental resolution $\sim 70 \mathrm{~km} \mathrm{~s}^{-1}$ in the vicinity of the $\mathrm{H} \alpha$ feature. The data span a large wavelength range of $3600 \AA$ to $10300 \AA$ which covers key spectral features from the optical to the near-IR crucial for measuring stellar populations and extracting both stellar and gas kinematics. Each galaxy in the survey is targeted with one of 17 IFU fibre bundles per plate, with the bundles containing $19(\times 2)$, $37(\times 4), 61(\times 4), 91(\times 2)$, or $127(\times 5)$ fibres (see Drory et al. 2015 , for further details). The size of the fibre bundle typically matched to the angular size of the galaxy, such that the majority of the targets have spectral coverage to at least $1.5 R_{\mathrm{e}}$. The fibres within the bundles have radius 2 arcsec, which matches the typical ground-based seeing at APO. During the observations, a three-point dither pattern is applied, such that the inter-fibre gaps in each IFU bundle are sampled (Law et al. 2015). The final reduced datacubes are resampled to square spaxels of size 0.5 arcsec with a median spatial resolution of $\sim 2.5$ arcsec FWHM (Law et al. 2016). The flux calibration of the data is better than 5 percent (Yan et al. 2016a) for 89 per cent of the wavelength range, and 1.7 per cent between $\mathrm{H} \beta$ and $\mathrm{H} \alpha$.

MaNGA has three main samples, the primary sample, the secondary sample, and the colour-enhanced sample, which are selected to meet several different science goals. The primary sample is designed such that $\sim 80$ per cent of the galaxies in this sample have IFU coverage to at least $1.5 R_{\mathrm{e}}$, and the sample has a mean redshift $\langle z\rangle=0.03$. The primary sample also includes a "colour-enhanced" sample, which contains galaxies selected to lie in underrepresented regions of the colour magnitude diagram, including highmass blue galaxies, "green valley" galaxies, and, importantly for this study, low-mass red galaxies. These colour-enhanced galaxies are also targeted to $1.5 R_{\mathrm{e}}$. The secondary sample is designed such that $\sim 80$ per cent of the galaxies have IFU coverage to at least $2.5 R_{\mathrm{e}}$, and as such, has a higher mean redshift than the primary sample. For further details of the survey design, see Yan et al. (2016b).

\subsubsection{Mid-Infrared Photometry}

We also use mid-infrared (IR) photometry in this work, which is not provided as part of the NASA Sloan Atlas. The mid-IR photometry used in this work is taken from the Wide Field Infrared Survey Explorer (WISE Wright et al. 2010) All Sky Survey. WISE is a mid-IR space based telescope, which imaged the whole sky in four photometric bands: $3.4 \mu \mathrm{m}$ (W1), $4.6 \mu \mathrm{m}$ (W2), $12 \mu \mathrm{m}$ (W3), and $22 \mu \mathrm{m}$. The $3.4 \mu \mathrm{m}$ and $4.6 \mu \mathrm{m}$ bands are sensitive to stellar light and hot dust respectively, making [3.4] - [4.6] colours ideal for the identification of certain types of AGN. The $12 \mu \mathrm{m}$ band traces crucial lines associated with recent star formation (e.g. and the line), and can thus be used to separate truly quenched galaxies from those with star formation in the past 2 Gyr. We matched the positions of the MaNGA low-mass galaxy sample to the WISE All Sky Survey photometry catalogue, and photometry was found for 303/310 of the low-mass galaxies we selected in Section 2.2. The WISE photometry is left in its native Vega magnitude system.

\subsection{Separating star forming and quenched galaxies}

The MaNGA survey targets galaxies with $10^{9} \mathrm{M}_{\odot} \lesssim M_{\star} \lesssim$ $5 \times 10^{11} \mathrm{M}_{\odot}$, and we select the lowest mass galaxies in the survey for this work. This builds on the sample of lowmass, quenched galaxies presented in Penny et al. (2016), which was selected from galaxies in SDSS Data Release 13 (DR13, Albereti et al., submitted). We first identify all low-mass, dwarf-like galaxies in SDSS-MaNGA with spectra reduced prior to June 2016 (known internally as MaNGA MPL-5), which were processed using Version 2.0.1 of the MaNGA Data Reduction Pipeline. This sample was released in SDSS data release 14 (DR14 Abolfathi et al. 2017). We identify dwarf galaxies as all galaxies with absolute magnitudes fainter than $M_{\mathrm{r}} \approx-19$, central stellar velocity dispersions $\sigma_{\star}<100 \mathrm{~km} \mathrm{~s}^{-1}$, and stellar mass $M_{\star}<5 \times 10^{9} \mathrm{M}_{\odot}$ $\left(M_{\star} \lesssim 2.5 \times 10^{9} \mathrm{M}_{\odot}\right.$ as listed in the NASA Sloan Atlas, which assume $h=1)$ ). 310 MaNGA galaxies meet these criteria. The galaxies in this sample have $-19.04<M_{\mathrm{r}}<-15.85$, and colours $0.64<(u-r)<3.59$. The median stellar mass of the sample is $2.3 \times 10^{9} \mathrm{M}_{\odot}$ assuming $h=0.7$, comparable with that of the Large Magellanic Cloud $\left(2.3 \times 10^{9} \mathrm{M}_{\odot}\right.$ James \& Ivory 2011). This is similar to the selection criteria used in Penny et al. (2016).

All spectra within $1 R_{\mathrm{e}}$ are stacked to provide a high signal-to-noise spectrum for each galaxy in our sample, and are provided as part of Version 2.0.2 of the MaNGA data analysis pipeline (DAP, Westfall et al., in prep). These binned spectra provide an ideal "first look" at the galaxy, allowing for the quick identification of the integrated properties of an individual galaxy, without having to examine the galaxy spaxel-by-spaxel. This combined spectrum will include any star forming regions within $1 R_{\mathrm{e}}$, which will be identified through the presence of emission in the stacked spectrum. A single object with extremely low $S / N=2$ is removed, as the resulting spectrum is unusable for our science analysis, with the remaining stacked spectra having $11<S / N<180$.

Emission-line strengths are provided as part of the MaNGA data analysis pipeline. First, as part of the DAP fit, the emission lines are masked, and the stellar continuum is modelled using the kinematic and stellar population fitting package pPXF (Cappellari \& Emsellem 2004). The stellar continuum model is constructed using a thinned version of the MILES spectral library, and due to the wavelength coverage of the library, the model spans the wavelength range $3525<\lambda<7500$. The model is also broadened to match the stellar velocity dispersion of the galaxy, and thus the absorption lines can be cleanly subtracted from the spectrum. The residual emission lines are then modelled using Gaussian profiles, with 21 different lines fit in total. The summed line fluxes, equivalent widths, and velocity dispersions are provided as part of the DAP output.

To build a sample of low-mass quenched galaxies, we must first remove any star-forming dwarfs using line strength diagnostics. We identify such galaxies using emission linestrength diagnostic methods, as colour alone cannot be used to accurately characterise a galaxy as quenched or star forming, as dusty star-forming galaxies will remain in the sample. However, no single line-strength diagnostic system can reliably class all galaxies as star forming, quenched, or host- 
ing AGN. For example, if the spectrum of a galaxy does not exhibit the $\mathrm{H} \beta$ feature in emission due to dust obscuration or weak emission, it cannot be placed on the (BPT Stasińska et al. 2008) diagram.

\subsubsection{WHAN classification}

To identify quenched, low-mass galaxy candidates from our sample of dwarf galaxies, we therefore use the width of $\mathrm{H} \alpha$ vs. [N $\mathrm{NI}$ ] line strength diagnostic (WHAN, Cid Fernandes et al. 2011). This diagram uses the line strength ratio of the two most prominent emission lines in most galaxy spectra: the $\mathrm{H} \alpha$ and $[\mathrm{N}$ II] $\lambda 6583$ lines. This classification scheme uses the equivalent width of the $\mathrm{H} \alpha$ line as a proxy for the ratio of the intrinsic $\mathrm{H} \alpha$ luminosity to the $\mathrm{H} \alpha$ luminosity expected due to stellar populations older than $10^{8} \mathrm{yr}$. This allows for the separation of emission due to star formation and AGN, from that originating from hot, evolved stars. The flux ratio between $[\mathrm{N}$ II] and $\mathrm{H} \alpha$ is used to separate AGN activity from star formation for galaxies with $\mathrm{EW}_{\mathrm{H} \alpha}>3 \AA$. The WHAN diagram also allows for the identification of galaxies in which the heating of their ionised gas is the result of old stars, rather than star formation or AGN activity, as well as those galaxies that cannot be classified via the BPT diagram due to absent absorption lines. For full details of the WHAN classification scheme, see Cid Fernandes et al. (2011).

First, star forming galaxies are removed from the sample. These galaxies have $\log ([\mathrm{N} \mathrm{II}] / \mathrm{H} \alpha)<-0.4$ and $\mathrm{EW}_{\mathrm{H} \alpha}>$ $3 \AA$ under the WHAN classification system, and 217 galaxies meet these criteria. 93 galaxies remain after the removal of star forming galaxies from our sample.

We also identify all emission-line free galaxies using the WHAN line-strength diagnostic method. These galaxies are identified by the absence of significant $\mathrm{H} \alpha$ and [N II] emission lines in their spectra, with both lines required to have equivalent widths $<0.5 \AA$, else no detectable $\mathrm{H} \alpha$ emission. 57 galaxies meet these criteria. We then identify all galaxies with weak $\mathrm{H} \alpha$ and [N $\mathrm{NI}$ ] lines, which are inconsistent with AGN activity- the "retired" galaxy populations in the Cid Fernandes et al. (2011) WHAN classification system. Their emission lines are the result of heating by hot, old, evolved, low-mass stars, and such objects have $0.5<E W_{\mathrm{H} \alpha}<3$. Fourteen low mass galaxies in our sample are "retired" objects, with emission line strengths inconsistent with ongoing star formation. The WHAN classification system therefore identifies 71 low-luminosity galaxies in MaNGA MPL5 that can be considered to be passively evolving.

Those galaxies classified as quenched with clear star formation or blue spiral morphologies are manually removed from the quenched galaxy sample. A visual inspection of its central spectrum revealed clear emission lines that were not properly measured by the DAP. This process removed 1 object from the quenched galaxy sample. We also remove one object at $z=0.278$ which had made our low-mass quenched galaxy sample. The NASA Sloan Atlas listed the redshift of this galaxy as $z=0.0286$, and the galaxy's absolute photometry was therefore incorrect. This process left 69 candidate galaxies that host little or no star formation. 33 objects in our quenched low-mass galaxy sample are drawn from the

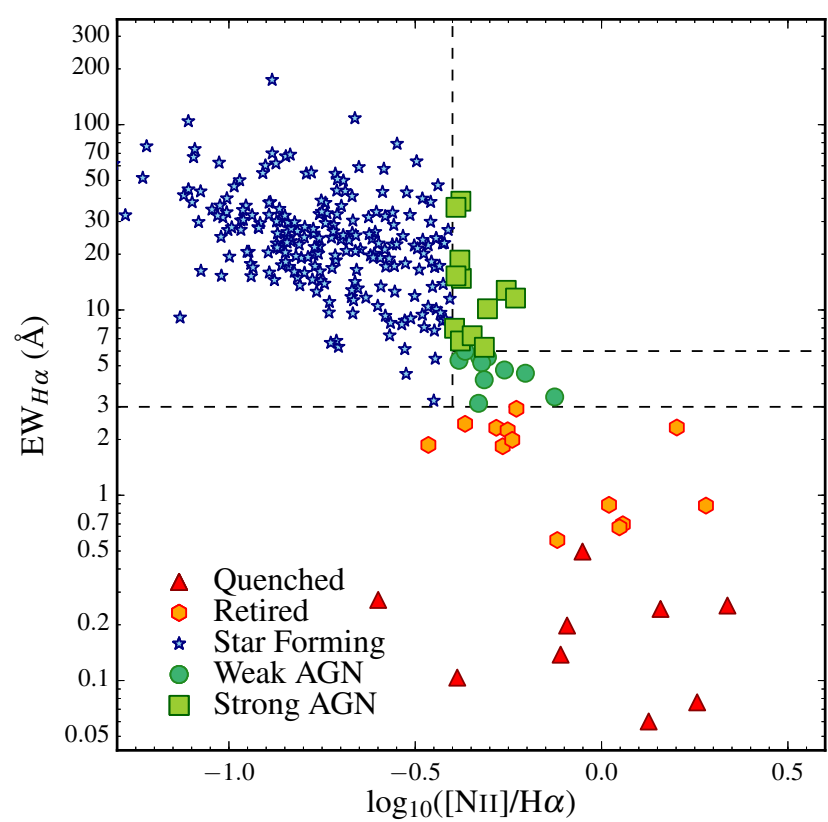

Figure 1. Equivalent width of $\mathrm{H} \alpha$ versus $[\mathrm{NII}] / \mathrm{H} \alpha$ (WHAN Cid Fernandes et al. 2011) diagram for faint $\left(\boldsymbol{M}_{\mathrm{r}}>-19\right)$ galaxies in the MaNGA survey. The dashed lines are the diagnostic lines from, which split the diagram into regions where the emission is dominated by star formation, AGN, or heating by hot, old stars. The majority of the galaxies $(217 / 310)$ below the luminosity cut of $M_{\mathrm{r}}>-19$ are star forming systems.

primary sample, 4 from the secondary sample, and 32 from the colour-enhanced sample.

The remaining 22 galaxies are classified as AGN on the WHAN diagram. The ten objects with $\mathrm{EW}_{\mathrm{H} \alpha}<6 \AA$ are weak AGN, in which the emission lines are likely the combination of both AGN and star formation activity. The remaining twelve galaxies have $\mathrm{EW}_{\mathrm{H} \alpha}>6 \AA$ classified as Seyfertlike emission. However, we note the majority of these are edge-on disk galaxies, and are likely heavily dust obscured. Nevertheless, we go on to examine the their spaxel-by-spaxel BPT diagrams.

\subsection{Local environment of quenched versus star forming low-mass galaxies}

We quantify the local environment of the low-mass quenched galaxies using both a neighbour method and a local galaxy density measure. First, we trace large-scale structure using a combination of the 2MASS redshift survey and SDSS. This redshift catalogue is complete to $M_{\mathrm{K}}=-23$ at $z=0.04$, comparable to the highest redshift of our MaNGA low-mass galaxy sample. Identical to (Penny et al. 2016), we find the bright neighbour galaxy with $M_{\mathrm{K}}<-23$ within $\pm 1000 \mathrm{~km} \mathrm{~s}^{-1}$ for each low-mass galaxy in our sample. We also count the number of bright galaxies with $M_{\mathrm{K}}<-23$ within a radius of $1.5 \mathrm{Mpc}$ (comparable to the virial radius of a galaxy cluster) and $\pm 1000 \mathrm{~km} \mathrm{~s}^{-1}$ of each low-mass galaxy.

The neighbour distances of our quenched and star forming samples as defined in Section 2.2 are shown in Fig. 2. For clarity, the WHAN-classified AGN are not included on this plot. A clear difference in environment is seen for 


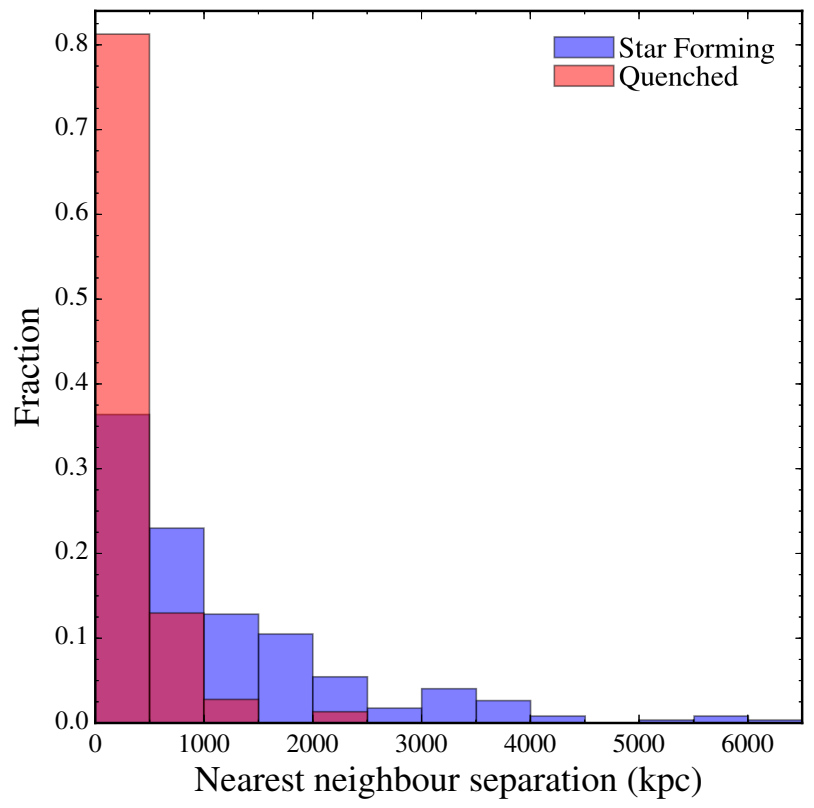

Figure 2. Histogram showing the separation of the low-mass galaxies from their nearest bright neighbour galaxy with $M_{\mathrm{K}}<$ -23 . The distribution for both the quenched and star forming low-mass galaxy samples as selected in Section 2.2 are shown. The distribution of the quenched galaxies are shown as red bars, and the star forming galaxies by blue bars. The purple regions are the overlap between the star forming and quenched objects.

the two samples, such that quenched galaxies are typically found at separations $<1000 \mathrm{kpc}$ from their bright neighbour galaxy. The low-mass, quenched galaxies have separations $17 \mathrm{kpc}<D_{\text {bright }}<4582 \mathrm{kpc}$ from their bright neighbour, with a median separation $228 \pm 72.8 \mathrm{kpc}$. In contrast, the purely star-forming objects have separations $32 \mathrm{kpc}<$ $D_{\text {bright }}<6056 \mathrm{kpc}$, with a median separation $706 \pm 78.5 \mathrm{kpc}$. A difference in local galaxy density is also seen, such that within a comoving radius of $1.5 \mathrm{Mpc}$ and a velocity separation $\pm 1000 \mathrm{~km} \mathrm{~s}^{-1}$, a quenched low-mass galaxy has a mean of $16 \pm 2.8$ bright neighbours with $M_{\mathrm{K}}<-23$, whereas a star forming low-mass galaxy has a mean $2.2 \pm 2.55$ bright neighbours using an identical magnitude limit.

\section{IONISED GAS IN QUENCHED GALAXIES}

We search for any objects in our low-mass, quenched galaxy sample that retain an ionised gas component. If a galaxy retains a gas reservoir, or if it is actively accreting gas from its surroundings, yet is no longer forming stars, then a feedback mechanism must be operational within that galaxy. By identifying those quenched galaxies that exhibit ionised gas, and quantifying their emission line flux ratios and the location within the galaxy of the emission, we can determine the heating mechanism acting upon the gaseous component.

Gas that is either co-rotating with the galaxy's stellar component, else offset by exactly $180^{\circ}$, has both components in dynamical equilibrium. However, when rotation of the ionised gas component is randomly offset, this is likely evidence of an accretion event. First, we identify all low mass galaxies with clear rotation in their $[\mathrm{O}$ III $] \lambda 5008, \mathrm{H} \alpha$, or
[N II] 26583 emission line features. These three lines are used as they are very sensitive tracers of ionisation, and typically the most prominent emission lines in a galaxy spectrum. The ratio between these features can be used to separate AGN heating from both star formation, and heating by old stellar populations (e.g. Cid-Fernandes et al., 2011). From our initial sample of 63 quenched dwarf galaxies, 12 are identified with clear rotation in both their stellar and ionised gas components. One object, MaNGA 1-38166, has a rotating ionised gas component, but no coherent stellar rotation. Excluding a single member of the Coma Cluster (MaNGA 1-456355), the quenched low-mass galaxies that retain an ionised gas component are found in galaxy groups with fewer than seven $L \star$ or brighter galaxies (see Section 2.3). Velocity maps showing the stellar and ionised gas velocity fields for each low-mass galaxy with clear rotation are shown in Fig. 3.

As can be easily seen in Fig 3 and Appendix A, the stars and ionised gas are not necessarily co-rotating, and thus not all of the galaxies are in dynamical equilibrium. We therefore go on to compare the kinematic position angles of the stellar and ionised gas components of these galaxies. The global kinematic position angles of the two components are measured using the method described in Appendix C of Krajnović et al. (2006). We note here that a number of the offset ionised gas velocity fields may be outflows (e.g. MaNGA 1-113520), so we use this measure only to check for gas that is neither co- or counter-rotating with the stellar component.

The average velocities of the $\mathrm{H} \alpha$ and $[\mathrm{N}$ II] $\lambda 6583$ lines are used in the calculation of the ionised gas kinematic PA, as they are typically among the strongest emission lines in the galaxy spectra. The velocities of the individual emission lines are output by the MaNGA-DAP, and are calculated using single-profile gaussian fits to the emission lines after the stellar continuum has been subtracted. The global kinematic PAs of the $\mathrm{H} \alpha$ and $[\mathrm{N} \mathrm{II}] \lambda 6583$ velocity fields typically agree to $<20^{\circ}$ for all objects. We set a range of $0^{\circ}$ to $360^{\circ}$ for the limits on the kinematic position angles to identify galaxies with counter-rotation between their stars and ionised gas.

Prior to the determination of the kinematic position angles, the stellar and ionised gas velocity maps are convolved with a gaussian filter to remove any noise which could affect the position angle measurements, especially for the galaxies targeted with the 19 fibre bundles. The filter size is chosen to be 2 spaxels, to remove artefacts on the scale of the noise from the velocity maps. For MaNGA 1-113520, which contains a counter-rotating core in its stellar component, we do not attempt to fit a single global kinematic position angle. Instead, we only fit the kinematic position angle for the centrally rotating component, with the outer component assigned a kinematic position angle offset by $180^{\circ}$ from the central region. We were unable to determine a stellar kinematic PA for MaNGA 1-38166, though a visual inspection of its stellar kinematic map suggests it hosts weak stellar rotation. The measured angles for both the stellar and gaseous components are given in Table1, along with their central stellar velocity dispersions and Sérsic indices.

For eight galaxies in our sample, the recovered kinematic position angles of both their stars and ionised gas have difference $\Delta \mathrm{PA}<30^{\circ}$ or $\Delta \mathrm{PA}>150^{\circ}$. These small kinematic offsets show the stars and gas within these objects are likely in dynamical equilibrium. However, clear differ- 

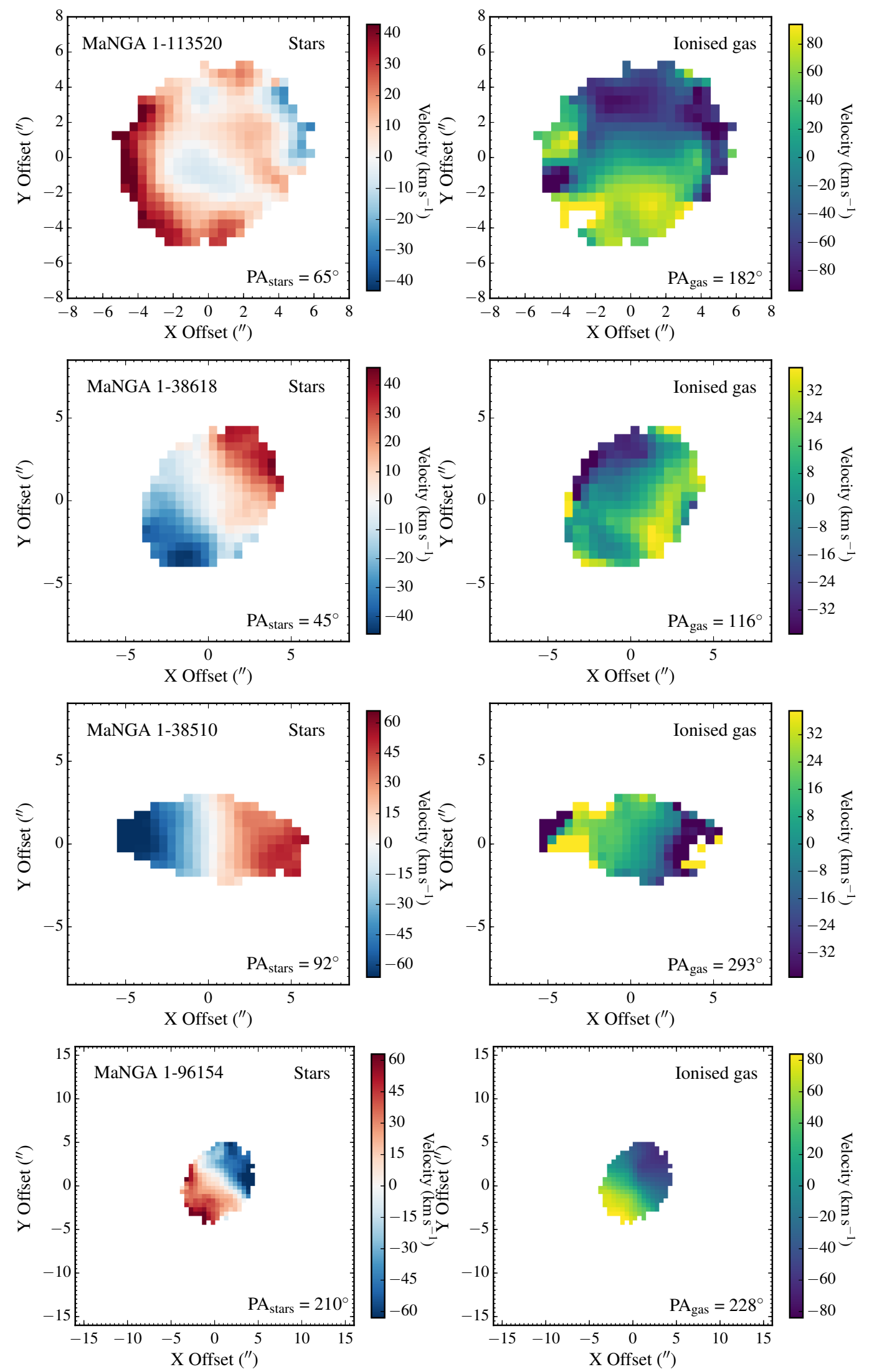

Figure 3. Example stellar velocity maps (left panel) and ionised gas velocity maps (right panel) for 4/14 MaNGA galaxies in our sample which contain an ionised gas component. We trace the ionised gas velocity feature using the $\mathrm{H} \alpha$ line. Two galaxies with co- or counter-rotating stellar and ionised gas components are shown, along with two galaxies that host kinematically offset stars and ionised gas. All have $M_{\mathrm{r}}>-19, M_{\star} \lesssim 5 \times 10^{9} \mathrm{M}_{\odot}$, and $\sigma_{\star}<100 \mathrm{~km} \mathrm{~s}^{-1}$, placing them in the dwarf galaxy regime. The remaining velocity maps are shown in Appendix A. 


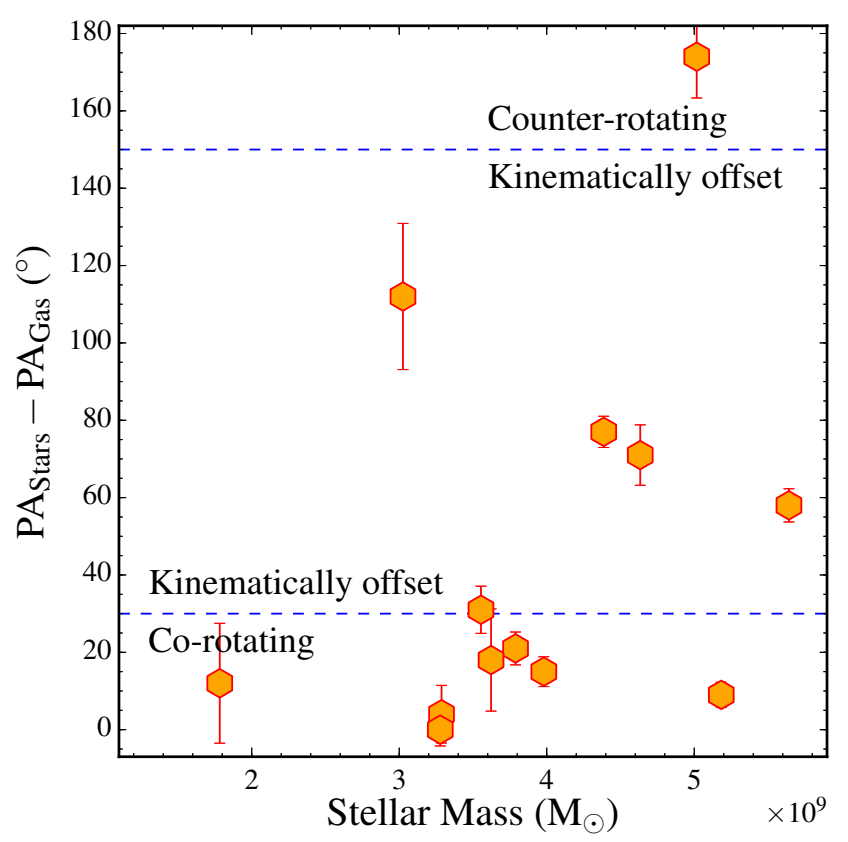

Figure 4. Difference between the stellar and the ionised gas kinematic position angles as a function of galaxy mass. Object with kinematic offsets $>180^{\circ}$ between their stellar and ionised gas components have been subtracted by $180^{\circ}$ for simplicity. No obvious trend for galaxies to host kinematically offset gas with stellar mass is seen. One object, MaNGA 1-38166 could not be included on this plot as we could not reliably measure a stellar kinematic position angle.

ences between the two kinematic position angles are seen for the remaining five galaxies, and these objects likely host recently accreted gas or satellites. A stellar kinematic position angle could not be determined for MaNGA 1-38166, though a visual inspection of the map suggests its stars and gas are co-rotating. In Fig. 4 , we plot $\triangle \mathrm{PA}$ as a function of galaxy stellar mass, and there is no preference for galaxies hosting kinematically offset gas to either higher or lower stellar masses. To characterise the heating mechanism of this ionised gas, we go on to examine the nature of their emission on the BPT diagram.

\subsection{Spaxel-by-spaxel BPT diagrams}

We construct BPT diagrams for all objects in our quenched sample which exhibit an ionised gas component, to separate those with nuclear starbursts from those exhibiting AGN activity. The line ratios are calculated for each spaxel in the datacube with a signal-to-noise $>10$ in the stellar continuum, and a signal-to-noise $>5$ in their $\mathrm{H} \alpha$ emission line. These emission line ratio diagrams are presented in Fig. 5 (for those objects with kinematically offset rotation), and Fig. 6 for those objects with corotating gas. Also plotted for diagnostic purposes are the Kewley et al. (2001) and Kauffmann et al. (2003) classification lines classification lines, which are used to separate extreme starbursts and H II regions from AGN-like emission. The Schawinski et al. (2007) division between Seyfert-like and LINER-like (Low Ionisation Nuclear Emission Region) emission line ratios is also plotted, to ensure we identify gen- uine AGN emission in these galaxies, rather than heating by hot, old stars, which can masquerade as LINER emission (e.g. Cid Fernandes et al. 2011; Belfiore et al. 2016). Each spaxel is coloured by its distance from the galaxy centre, such that central spaxels are represented by darker points.

Fig. 5 and Appendix B1 shows that the galaxies with kinematically offset gas exhibit AGN-like emission in their central regions, with no spaxels in any of the six galaxies consistent with current star formation. In contrast, eight of the nine galaxies plotted in Fig. 6 and Appendix C1 with co-rotating or counter-rotating ionised gas have spaxel-byspaxel BPT diagrams dominated by star formation or composite (AGN+star-formation) emission line ratios. Only one object with co-rotating stars and gas, MaNGA-1-230177, has clear AGN-like emission at its centre.

\subsubsection{WHAN-classified AGN}

We also construct spaxel-by-spaxel BPT diagrams for the 22 galaxies classed as AGN using the WHAN classification system, but we do not include these plots. 12 objects in this sample exhibit central spaxels in the AGN/LINER/star formation composite region of the BPT diagram, five of which do not exhibit any purely star forming spaxels, while seven host star formation in their outer regions. As such, these seven objects are not truly quenched galaxies, and have either blue colours $(u-r)<1.65$, else are dust reddened edgeon spirals with axial ratios $b / a \leq 0.4$. Ten galaxies have line ratios at their centres consistent with active star formation. As we cannot classify any of these objects as clear AGN host candidates, we do not analyse them further in this work.

\subsection{Emission-line maps}

Cheung et al. (2016) identified a number of galaxies in the MaNGA survey with AGN-powered maintenance-mode feedback, in which further star formation is prevented by the heating or removal of gas after the initial quenching episode. Such galaxies exhibit bi-symmetric emission line features, traced by the equivalent widths of their $\mathrm{H} \alpha$ line. These emission features are co-aligned with their ionised-gas velocity fields, and are found in $\sim 10 \%$ of MaNGA galaxies with stellar masses $\sim 2 \times 10^{10} \mathrm{M}_{\odot}$.

We construct maps showing the distribution of the $\mathrm{H} \alpha$ emission within the galaxy to search for such features in the low-mass galaxies with AGN-like emission. The equivalent widths for the $\mathrm{H} \alpha$ line are provided as part of the DAP output, and these maps are shown in Fig. 7. As can be seem in Fig. 7, four of the galaxies exhibit clear bi-symmetric emission features, though these features are not necessarily aligned with the velocity fields of their ionised gas. We also construct these maps for the galaxies with line ratios consistent with star formation, and these bi-symmetric features are not present in that sample.

\subsection{Mid-IR colours}

Mid-IR photometry is a useful diagnostic tool for the identification of AGN in the absence of radio or X-ray data, particularly for objects hosting Seyfert-like emission, or obscured AGN. For example, radio-loud AGN are expected to radiate 
Table 1. Basic properties for the low-mass galaxies identified as quenched galaxies in Section 3 which host an ionised gas component. Global kinematic position angles for their stellar $\left(\mathrm{PA}_{\star}\right)$ and ionised gas $\left(\mathrm{PA}_{\text {gas }}\right)$ components, along with the difference between the two values $\triangle \mathrm{PA}$ are provided. A global stellar kinematic PA could not be reliably measured for MaNGA 1-113520 due to the presence of a counter-rotating component.

\begin{tabular}{lccccccccccc}
\hline MaNGA-ID & Plate & IFU & $\begin{array}{c}\text { RA } \\
(\mathrm{J} 2000.0)\end{array}$ & $\begin{array}{c}\text { Dec } \\
(\mathrm{J} 2000.0)\end{array}$ & $z$ & $\begin{array}{c}M_{\mathrm{r}} \\
(\mathrm{mag})\end{array}$ & Sérsic $n$ & $\begin{array}{c}\sigma_{\star} \\
\left(\mathrm{km} \mathrm{s}^{-1}\right)\end{array}$ & $\begin{array}{c}\mathrm{PA}_{\text {stars }} \\
\left({ }^{\circ}\right)\end{array}$ & $\begin{array}{c}\mathrm{PA}_{\text {gas }} \\
\left({ }^{\circ}\right)\end{array}$ & $\begin{array}{c}\Delta \mathrm{PA} \\
\left({ }^{\circ}\right)\end{array}$ \\
\hline $1-38166$ & 8081 & 3702 & $03: 19: 47.24$ & $+00: 37: 25.8$ & 0.025 & -18.65 & 3.4 & $\ldots$ & $\ldots$ & $86 \pm 7.6$ & $\ldots$ \\
$1-38618$ & 8084 & 1902 & $03: 30: 29.42$ & $-00: 29: 19.6$ & 0.022 & -18.84 & 2.7 & 43 & $45 \pm 6.5$ & $116 \pm 9.4$ & 71 \\
$1-38510$ & 8155 & 1901 & $03: 32: 05.59$ & $+00: 28: 45.8$ & 0.022 & -18.73 & 1.6 & $\ldots$ & $92 \pm 1.5$ & $113 \pm 7.9$ & 21 \\
$1-379255$ & 8711 & 1901 & $07: 53: 03.98$ & $+52: 44: 35.5$ & 0.018 & -18.36 & 4.7 & 78 & $134 \pm 18.4$ & $22 \pm 9.2$ & 112 \\
$1-230177$ & 8942 & 6101 & $08: 19: 35.49$ & $+26: 21: 45.6$ & 0.020 & -18.82 & 1.8 & $\ldots$ & $37 \pm 2.2$ & $52 \pm 5.0$ & 15 \\
$1-488575$ & 8449 & 1902 & $11: 13: 49.70$ & $+22: 48: 38.4$ & 0.022 & -18.46 & 2.2 & $\ldots$ & $67 \pm 6.6$ & $71 \pm 6.0$ & 4 \\
$1-456355$ & 8931 & 6104 & $12: 57: 11.96$ & $+27: 06: 12.0$ & 0.025 & -19.02 & 1.6 & $\ldots$ & $167 \pm 2.7$ & $176 \pm 1.7$ & 9 \\
$1-284335$ & 8318 & 6103 & $13: 07: 17.01$ & $+45: 43: 41.3$ & 0.035 & -19.04 & 4.0 & $\ldots$ & $207 \pm 9.7$ & $21 \pm 9.9$ & 186 \\
$1-93551$ & 8483 & 1901 & $16: 25: 14.66$ & $+48: 43: 16.8$ & 0.021 & -17.86 & 6.0 & 49 & $101 \pm 14.9$ & $113 \pm 9.1$ & 12 \\
$1-96154$ & 8612 & 9102 & $16: 57: 35.35$ & $+39: 17: 09.9$ & 0.033 & -18.87 & 1.0 & $\ldots$ & $210 \pm 12.1$ & $228 \pm 14.0$ & 18 \\
$1-136305$ & 8606 & 3704 & $17: 03: 39.73$ & $+36: 23: 05.8$ & 0.025 & -18.45 & 1.1 & 56 & $278 \pm 2.6$ & $278 \pm 5.5$ & 0 \\
$1-178823$ & 8623 & 9102 & $20: 47: 03.31$ & $+00: 26: 12.4$ & 0.013 & -18.92 & 2.0 & $\ldots$ & $97 \pm 1.7$ & $354 \pm 6.7$ & 257 \\
$1-113520 *$ & 7815 & 1901 & $21: 10: 00.53$ & $+11: 30: 38.3$ & 0.017 & -18.98 & 2.3 & 52 & $65 \pm 4.5 *$ & $182 \pm 1.8$ & 58 \\
$1-29809$ & 8655 & 1902 & $23: 53: 52.52$ & $-00: 05: 55.4$ & 0.022 & -18.72 & 1.8 & $\ldots$ & $21 \pm 4.4$ & $232 \pm 8.9$ & 211 \\
\hline
\end{tabular}

*MaNGA 1-113520 hosts a counter-rotating core, and thus the measured kinematic position angle is for the core region only.
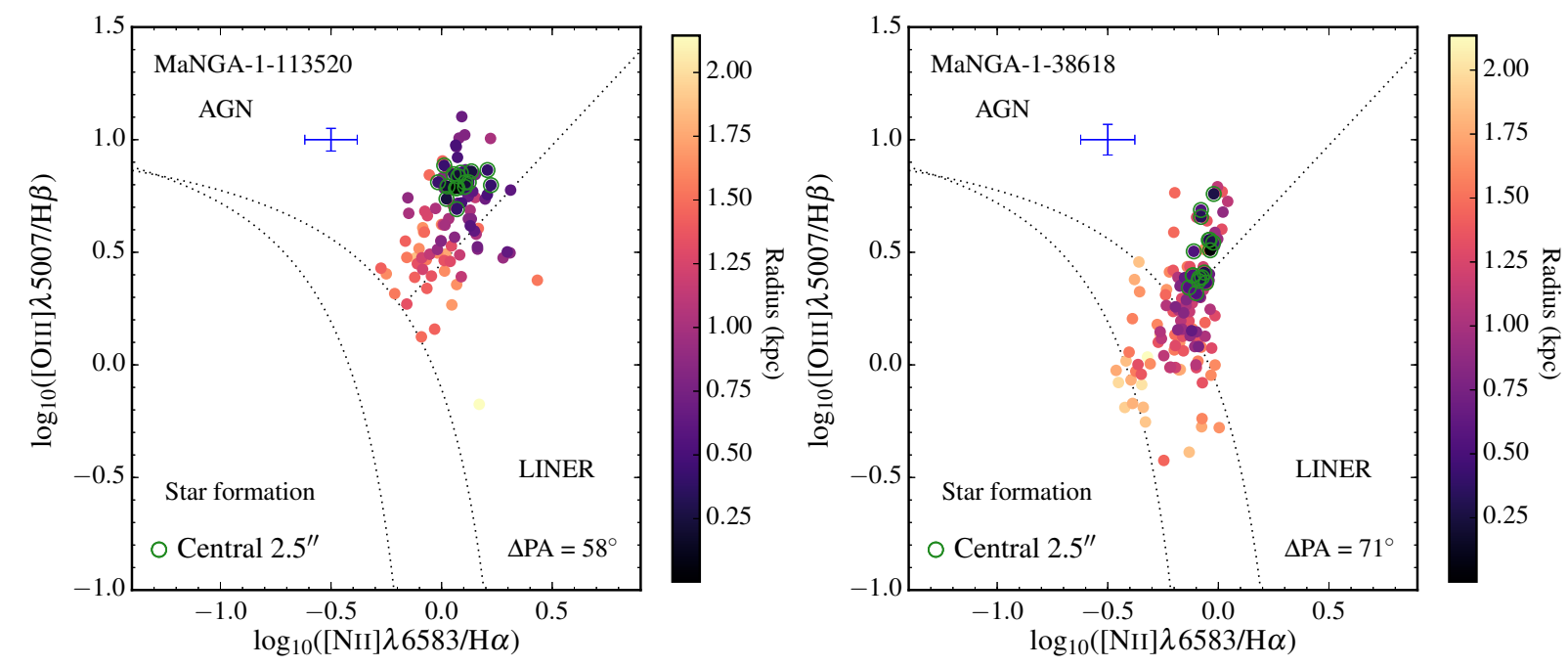

Figure 5. Spaxel-by-spaxel BPT emission line ratio diagrams for two of the five galaxies in our sample with a kinematically-offset gas component. BPT diagrams for the remaining two galaxies are given in Appendix B1 The colour of each point corresponds to its distance from the galaxy centre, with the darkest points representing the central spaxels. Spaxels in the central 2.5 arcsecs (the PSF of the reduced datacubes) are circled in green. No object in this sample exhibits line ratios consistent with solely being from ongoing star formation. Also plotted for diagnostic purposes are the Kewley et al. (2001) and Kauffmann et al. (2003) classification lines, which are used to separate extreme starbursts and H II regions from AGN-like emission. The Schawinski et al. (2007) division between Seyfert-like and LINER-like (Low Ionisation Nuclear Emission Region) emission line ratios is also plotted.

brightly in the mid-IR if the dust torus of the AGN strongly obscures the central black hole. Low-excitation radio galaxies hosting AGN are harder to identify in the mid-IR however, and exhibit a range of mid-IR colour (e.g. Gürkan et al. 2014).

Mid-IR colours can also be used to separate out quenched stellar populations from those with recent star formation. For example, red-sequence, optically quenched galaxies that have undergone star formation in the past 2 Gyr exhibit excess mid-IR emission (e.g. Ko et al. 2013). This is due to emission from the dusty circumstellar en- velopes of asymptotic giant branch (AGB) stars, and polycyclic aromatic hydrocarbon $(\mathrm{PAH})$ release from carbon stars. The contribution of these processes to the mid-IR emission of a galaxy decreases as the mean stellar age of the galaxy increases.

We confirm the current star formation state of the galaxies using mid-IR photometry taken from the WISE telescope (Wright et al. 2010) All Sky Survey. We plot the [3.4] - [4.6] versus [4.6] - [12] colours of the entire low-mass galaxy sample in Fig. 8. Those objects with $S / N<2$ in the WISE are shown as unfilled circles. We also plot the posi- 

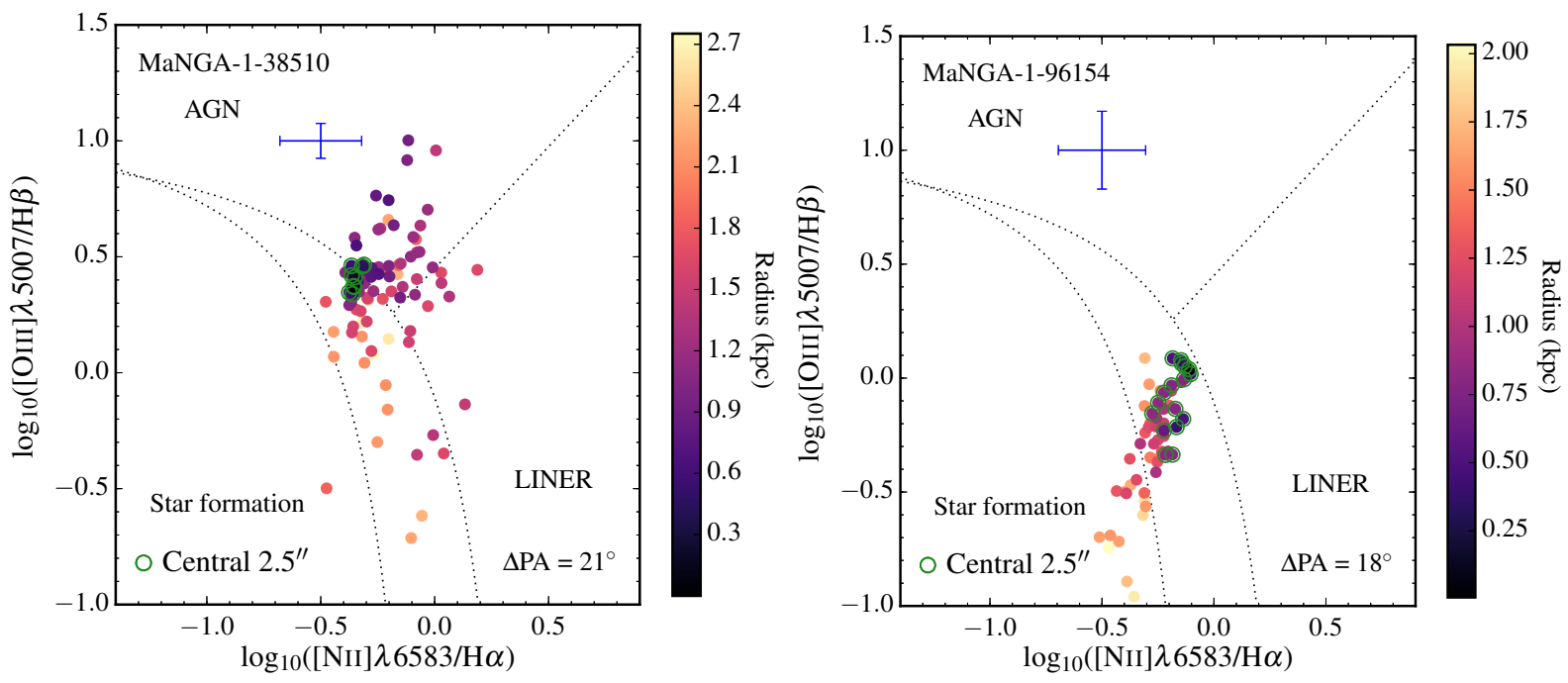

Figure 6. Spaxel-by-spaxel BPT emission-line ratio diagrams for two galaxies in our sample with gas that is either co-rotating or offset by $\approx 180^{\circ}$ (counter rotating) from their stellar component. BPT diagrams for the remaining seven galaxies are given in Appendix C1. The colour of each point corresponds to its separation from the centre of the galaxy in arcseconds. Spaxels in the central 2.5 arcsecs (the PSF of the reduced datacubes) are circled in green. The majority (8/9) of the galaxies with co- or counter-rotating gas have emission line ratios consistent with star formation or composite AGN/star formation. The remaining galaxy, MaNGA-1-230177, has central spaxels that exhibit emission line ratios consistent with AGN activity.

tions of the objects classed as AGN or star forming via the line-strength diagnostic techniques presented in Section 3.1. One object in our AGN sample, MaNGA 1-38618, is excluded from this plot, as its photometry is flagged as unreliable by the WISE photometry pipeline, likely due to its close proximity to a foreground star (WISE has an angular resolution of $\sim \operatorname{arcsec}$ ).

One galaxy, MaNGA 1-29809, lies in the region of the WISE [3.4] - [4.6] versus [4.6] - [12] colour-colour diagram typically occupied by Seyfert galaxies and obscured AGN (see figure 12 of Wright et al. 2010), with [4.6] - [12] = 3.50, and [3.4] $-[4.6]=1.83$. The red [3.4] $-[4.6]$ colour indicates a heavily obscured Type-2 AGN (Jarrett et al. 2011). The remaining BPT-classified AGN have $1.19<[4.6]-[12]<1.73$, consistent with them hosting little or no star formation, and they likely host primarily quenched stellar populations. Never-the-less, they are not as blue as many elliptical galaxies, which typically have $0<[4.6]-[12]<1.5$. In contrast, those galaxies which exhibit composite or starformation like emission line ratios are typically redder, with $1.72<[4.6]-[12]<2.62$, and lie in the region of the colourcolour diagram occupied by star forming galaxies. [4.6]-[12] colours indicate the amount of dust heating in a galaxy due to star formation, with bluer colours due to little-to-no star formation. Though we do not plot them in Fig. 8 for simplicity, those galaxies identified as AGN using the WHAN classification system have mid-IR [4.6] - [12] colours consistent with active star formation.

\section{DISCUSSION}

\subsection{Low-mass AGN-host galaxies}

We identify six low-mass, non-star forming galaxies that exhibit AGN-like emission line ratios in their spatially-resolved
BPT diagrams (Figs. 5 and 6). Of these, six have kinematically offset gas, such that the velocity field of their ionised gas component is offset by $>30^{\circ}$ from the kinematic PA of their stellar component (Fig. 3). The remaining galaxy with central AGN-like emission has an ionised gas component that is co-rotating with the stars. All six candidates have $M_{\star} \lesssim 5 \times 10^{9} \mathrm{M}_{\odot}$, and $M_{r}>-19$, placing them in the dwarf galaxy regime.

Based on the kinematic offset of the gas in the majority of the dwarfs with nuclear AGN-like emission, and the large velocity offset from the stellar kinematics, we infer that this gas is either being accreted onto, or expelled from, the low-mass galaxies. As these low-mass galaxies also exhibit AGN-like emission line ratios, we suggest that maintenancemode AGN feedback is preventing the gas in these galaxies from cooling and triggering new bursts of star formation. These faint galaxies are therefore likely low-mass analogues of the "red geysers" presented in (Cheung et al. 2016), which maintain quiescence through low-level AGN heating which prevents accreted gas from cooling and forming new stars. Two galaxies in particular, MaNGA 1-113520 and MaNGA 1-230177, do not contain any spaxels in their MaNGA datacube with emission line ratios consistent with photoionisation from star forming galaxies. We discuss the properties of individual galaxies in the sample with evidence for AGN feedback below.

\subsubsection{MaNGA 1-38618}

MaNGA 1-38618 has a stellar mass $2.8 \times 10^{9} \mathrm{M}_{\odot}$, and $M_{r}=$ -18.65. It exhibits weak $\mathrm{H} \alpha$ emission throughout its structure, with no clear symmetric features and a kinematic position angle offset between its gas and stars of $\sim 90^{\circ}$. It has emission line ratios in its central regions consistent with 

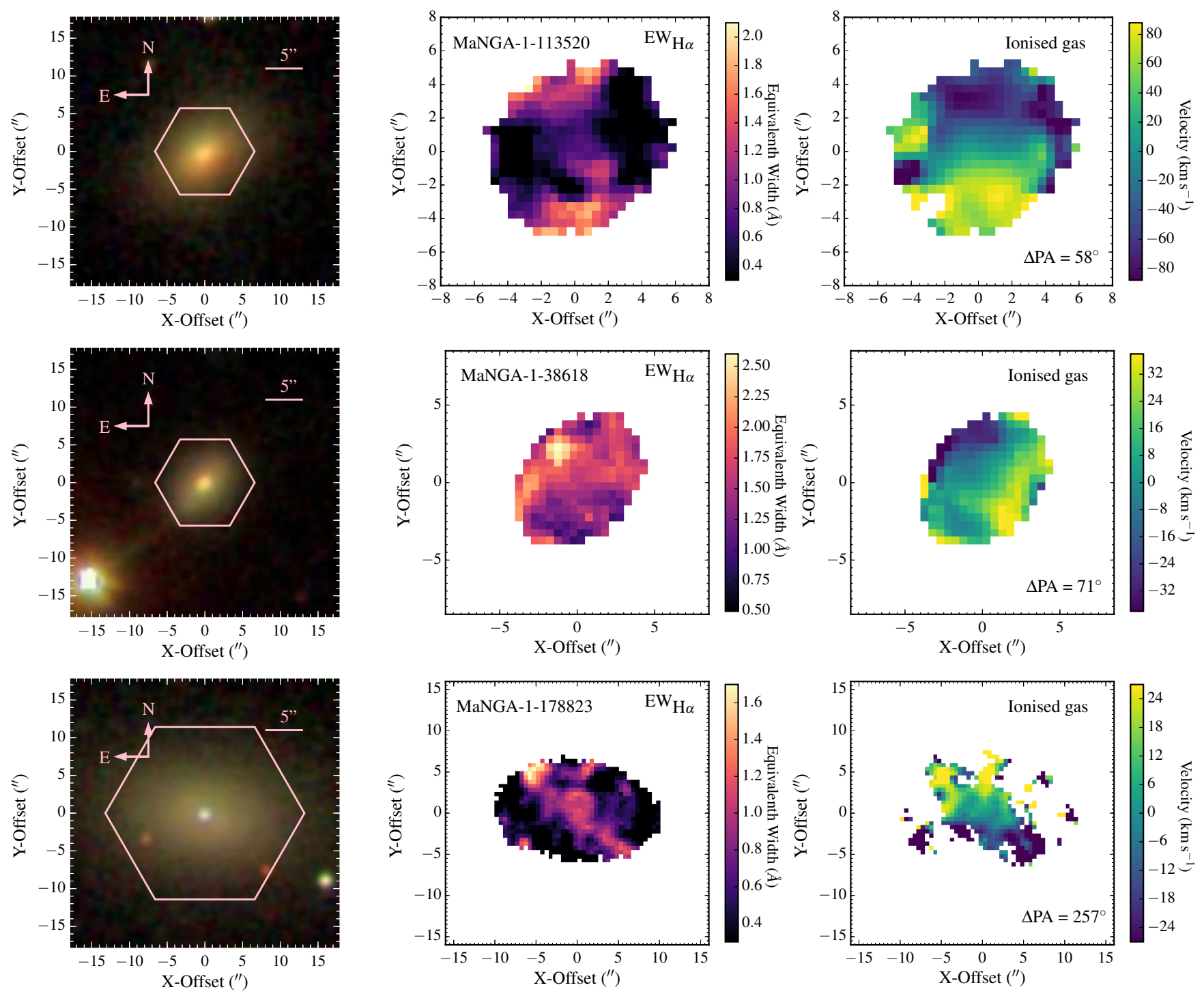

Figure 7. H $\alpha$ equivalent maps for the six dwarfs in which AGN-like emission dominates at their centres. Their ionised gas velocity fields are shown for comparison.

AGN/LINER activity, while at larger radii the emission is composite between star formation and LINER-like.

\subsubsection{MaNGA 1-379255}

MaNGA 1-379255 has a stellar mass of $3.03 \times 10^{9} \mathrm{M}_{\odot}$. The galaxy has a kinematic offset of $77^{\circ}$ between its gas and stars, and contains central spaxels which lie on the region of the BPT diagram occupied by AGN-like galaxies. Its emission features are not bi-symmetric. However, it has few spaxels above our signal-to-noise threshold.

\subsubsection{MaNGA 1-230177}

MaNGA 1-230177 is unusual in our sample of six dwarf-like AGN hosts, in that its gaseous and stellar components are co-rotating, with a kinematic offset of $15^{\circ}$ between the two. It has a stellar mass $3.98 \times 10^{9} \mathrm{M}_{\odot}$, and it has bar-like in shape with no spiral arms in gri colour imaging. The galaxy also exhibits very clear bi-symmetric $\mathrm{H} \alpha$ emission aligned with its minor axis.

\subsubsection{MaNGA 1-178823}

MaNGA 1-178823 is the lowest redshift object in our AGN sample, with $z=0.013(48 \mathrm{Mpc})$. It has a stellar mass $4.39 \times 10^{9} \mathrm{M}_{\odot}$, and $M_{r}=-18.92$. MaNGA 1-178823 has a bright, point-like nucleus clearly visible in its SDSS colour imaging, consistent with emission from an AGN, or the nuclear star clusters seen in bright dwarf galaxies in galaxy clusters like Fornax (e.g. Muñoz et al. 2015). It has very obvious bi-symmetric emission, and like the majority of galaxies in our AGN sample, it exhibits a large kinematic offset between its gas and stars, such that the rotation of its ionised gas emission as traced by the $\mathrm{H} \alpha$ emission line is offset by $61^{\circ}$ from its stellar component. It also exhibits weak $D 4000$ absorption at its centre (i.e. younger mean stellar ages) compared to outer regions. MaNGA 1-178823 is located in the 

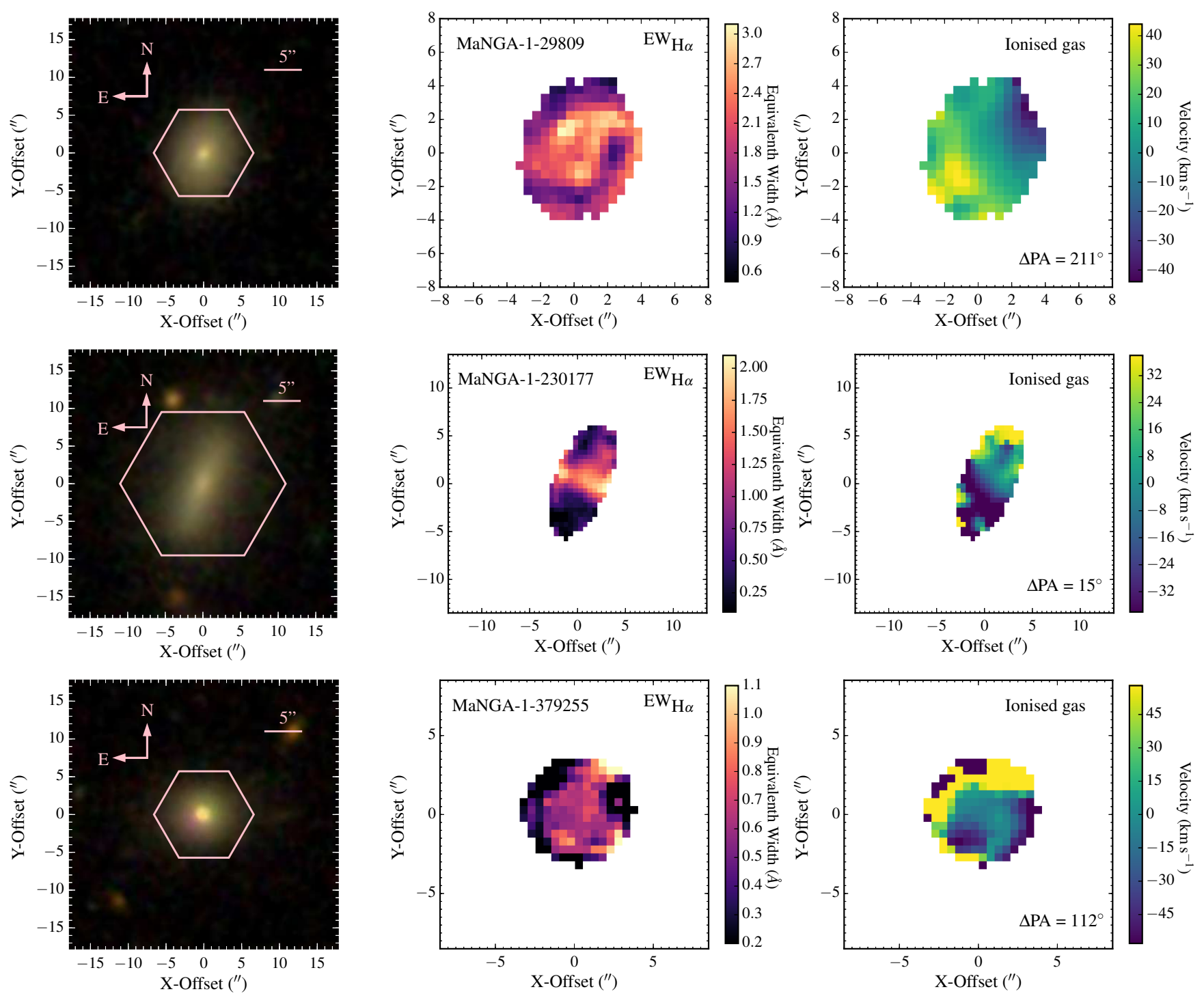

Figure 7 - continued $\mathrm{H} \alpha$ equivalent maps for the six dwarfs in which AGN-like emission dominates at their centres.

NGC 6962 group, at a projected distance of just $16 \mathrm{kpc}$ and $122 \mathrm{~km} \mathrm{~s}^{-1}$ from the S0 galaxy NGC 6959, and the two galaxies are therefore likely tidally interacting. The BPT diagram of the galaxy is primarily dominated by LINER-like emission to large radii, suggesting the presence of old, low-mass stars, though the nuclear region exhibits Seyfert-like emission. Based on line-strength ratios, it does not exhibit any star formation throughout its structure.

\subsubsection{MaNGA 1-113520}

MaNGA 1-113520 does not exhibit any emission lines in the star forming part of the BPT diagram, with the most central spaxels exhibiting AGN emission line ratios. The galaxy exhibits a counter-rotating core in its stellar component, and its gas is kinematically offset from the stellar components by $\sim 68^{\circ}$. It furthermore exhibits bi-symmetric $\mathrm{H} \alpha$ emission, with this emission spatially aligned with the kinematicallyoffset gas component 7 . The equivalent widths of the $\mathrm{H} \alpha$ features increase with radius from the galaxy nucleus, and resembles several cartoon models of AGN feedback. It is not detected in MaNGA Green Bank H i single dish follow-up observations (Masters et al., in prep.). The galaxy is not detected at either radio or X-ray wavelengths, and has mid-IR colours [3.4] $-[4.6]=1.6$ and [4.6] $-[12]=-0.0$, consistent with the galaxy hosting little star formation in the past 2 Gyr.

\subsubsection{MaNGA 1-29809}

Of the six galaxies with AGN-like emission, one galaxy, MaNGA 1-29809 shows evidence across multiple wavelengths for hosting an active nucleus. The strongest $\mathrm{H} \alpha$ emission is centrally located, with a hint of a ring-like structure at larger radii. It has WISE mid-IR colours [3.4]-[4.6] = 1.8 and [4.6] $-[12]=3.5$, placing it in the region of the colourcolour diagram occupied by Seyferts, ULIRGS, and LINERS. This object was also identified as a dwarf AGN by Sartori et al. (2015) based on this [3.4]-[4.6] mid-IR colour. It is offset from a point-like source in the radio-continuum 


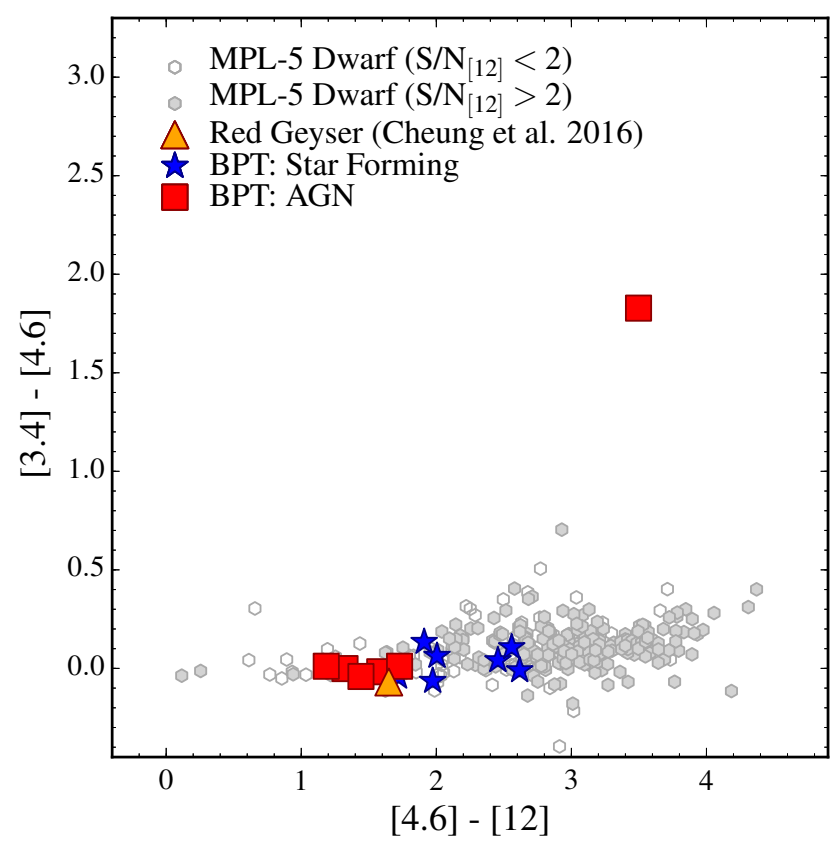

Figure 8. WISE colour-colour diagram for low-mass galaxies in MaNGA. The BPT-classified AGN hosts are shown as red squares. In general, the AGN-hosts exhibit bluer [4.6]-[12] colours than those that host composite or star forming regions at their centres, consistent with little-to-no ongoing star formation. However, one low-mass galaxy, MaNGA-MaNGA 1-29809, has an extremely red[3.4]-[4.6] colour, consistent with those of Seyfert-like hosts previously examined in the literature (e.g. Gürkan et al. 2014).

Faint Images of the Radio Sky at Twenty Centimeters (FIRST) survey (Becker et al. 1995) by 30", with a flux $3.19 \pm 0.097 \mathrm{mJy}$. No obvious galaxy or star is detected at optical wavelengths is detected at this location. No X-ray observations are available for this galaxy.

With a stellar mass $3.6 \times 10^{9} \mathrm{M}_{\odot}$, it is one of the lowest mass galaxies with clear signatures of hosting an AGN identified to date. MaNGA 1-29809 is fairly well isolated, located at a distance $D=928 \mathrm{kpc}$ and $\Delta v=799 \mathrm{~km} \mathrm{~s}^{-1}$ from the S0 galaxy IC $1517\left(M_{K}=-24.6\right)$. The central velocity dispersion of MaNGA 1-29809 is well below the BOSS spectrograph's instrumental resolution, and thus we cannot easily calculate the mass of its supermassive black hole using the central velocity dispersion. However, we can place limits on this mass, using $\sigma_{\text {cen }}=50 \mathrm{~km} \mathrm{~s}^{-1}$ as an upper limit on the galaxy's central velocity dispersion. With a central velocity dispersion $\sigma_{\text {cen }}=50 \mathrm{~km} \mathrm{~s}^{-1}$, this would imply a central black hole mass $<10^{6} \mathrm{M}_{\odot}$, assuming MaNGA 1-29809 lies on the $M-\sigma$ relation (e.g. Ferrarese \& Merritt 2000). However, the resolution of the data are not sufficient to examine the broad line regions of the AGN emission.

\subsection{Low-mass galaxies hosting star formation}

The remaining eight galaxies in our sample do not exhibit emission line features consistent with AGN activity. Instead, their spatially resolved BPT diagrams are dominated by line ratios suggesting they host ongoing star formation. This star formation is typically located in the outer regions of the galaxies, and most ( 7 out of 8 ) have central spaxels that reside in the star-formation/AGN/LINER composite region of the BPT diagram, i.e the region between the Kewley et al. (2001) and Kauffmann et al. (2003) diagnostic lines.

\subsection{Alternative heating mechanisms}

AGN-like emission line ratios do not necessarily mean the gas in that galaxy has been ionised by a central supermassive black hole. Several other heating mechanisms exist within galaxies that can result in such ratios, including heating by hot, old, evolved stars (e.g. post-AGB stars). Belfiore et al. (2016) demonstrated that extended LINERlike emission found in many MaNGA galaxies is likely the result of ionisation by post-AGB stars. Post-AGB stars are the main source of ionising photons upon the cessation of star formation (Stasińska et al. 2008; Cid Fernandes et al. 2011), though it is unclear if post-AGB stars are able to power the observed equivalent widths of LINER-like host galaxies due to the difficulty in modelling post-AGB stars. Belfiore et al. (2016) refer to galaxies with LINER-like linestrength ratios resulting from heating by old stars as LIERs, as they masquerade as LINERS on the BPT diagram. However, the AGN-candidates in our sample have Seyfert-like emission at their centres, unlike that seen in the the LIER galaxies presented in Belfiore et al. (2016).

The mid-IR colours of all but one of the BPT-classified AGN are blue ([4.6] - [12] < 1.7), showing a lack of recent star formation throughout their structures. These line ratios might therefore be the result of feedback from hot, evolved stars. We have assumed in this work that gas is either being driven in or out of the galaxies, due to the different kinematic properties of the stellar and ionised gas components. This kinematic offset shows the gas is not in dynamical equilibrium with the rest of the galaxy. Furthermore, several of the galaxies exhibit elevated $\mathrm{H} \alpha$ velocity dispersions along their bi-symmetric emission line features, indicating gas outflows. However, higher spatial and instrumental resolution IFU data is needed to fully examine the nature of this emission.

\section{CONCLUSIONS}

We present evidence for AGN feedback in low-mass galaxies. Our sample, which is drawn from the first 2 years of the SDSS-IV MaNGA survey, provides spatially resolved conformation that low-mass galaxies which exhibit evidence for gas inflow/outflow are prevented from forming stars via AGN feedback. These are likely low-mass analogues of the "red geysers" presented in (Cheung et al. 2016). This result suggests that AGN feedback may play an important feedback role in low mass galaxies with $M_{\star} \sim 10^{9} \mathrm{M}_{\odot}$. This feedback is likely most important in the galaxy group environment in order to stop dEs with $M_{\star} \sim 10^{9} \mathrm{M}_{\odot}$ undergoing further star formation after an initial quenching episode through e.g. tidal interactions. We summarise our conclusions below.

- A key result of these study is that maintenance-mode AGN feedback may be required for bright dE galaxies to maintain quiescence in galaxy groups and the outskirts of clusters. 
- Five low-mass galaxies in our sample exhibit ionised gas components as traced through the $\mathrm{H} \alpha$ emission line that are kinematically offset by $\gtrsim 30^{\circ}$ from their stellar velocity field. This means the gas in these galaxies is not in dynamical equilibrium, and it is either a recently accreted component, else an outflow.

- All five of galaxies with kinematically offset gas exhibit AGN-like emission line ratios at their centres, along with one galaxy that has an ionised gas component that is co-rotating with its stars.

- One low-mass galaxy, MaNGA 1-29809, exhibits evidence at optical and mid-IR wavelengths for hosting an AGN with Seyfert-like emission line ratios. With a stellar mass $3.6 \times 10^{9} \mathrm{M}_{\odot}$, this is one of the lowest-mass AGN host galaxies identified to date. This makes it an ideal candidate for multi-wavelength follow-up observations, including both high-resolution imaging and more detailed IFU spectroscopy.

While it is usually assumed that AGN feedback is unimportant in low-mass galaxy evolution, we have demonstrated here that $\sim 10$ per cent of galaxies in MaNGA with stellar masses $\lesssim 5 \times 10^{9} \mathrm{M}_{\odot}$ are AGN host candidates. Detailed observational followup is therefore needed to fully understand the role of feedback in these galaxies.

\section{ACKNOWLEDGEMENTS}

Funding for the Sloan Digital Sky Survey IV has been provided by the Alfred P. Sloan Foundation, the U.S. Department of Energy Office of Science, and the Participating Institutions. SDSS acknowledges support and resources from the Center for High-Performance Computing at the University of Utah. The SDSS web site is www.sdss.org.

SDSS is managed by the Astrophysical Research Consortium for the Participating Institutions of the SDSS Collaboration including the Brazilian Participation Group, the Carnegie Institution for Science, Carnegie Mellon University, the Chilean Participation Group, the French Participation Group, Harvard-Smithsonian Center for Astrophysics, Instituto de Astrofísica de Canarias, The Johns Hopkins University, Kavli Institute for the Physics and Mathematics of the Universe (IPMU) / University of Tokyo, Lawrence Berkeley National Laboratory, Leibniz Institut für Astrophysik Potsdam (AIP), Max-Planck-Institut für Astronomie (MPIA Heidelberg), Max-Planck-Institut für Astrophysik (MPA Garching), Max-Planck-Institut für Extraterrestrische Physik (MPE), National Astronomical Observatories of China, New Mexico State University, New York University, University of Notre Dame, Observatório Nacional / MCTI, The Ohio State University, Pennsylvania State University, Shanghai Astronomical Observatory, United Kingdom Participation Group, Universidad Nacional Autónoma de México, University of Arizona, University of Colorado Boulder, University of Oxford, University of Portsmouth, University of Utah, University of Virginia, University of Washington, University of Wisconsin, Vanderbilt University, and Yale University.

This research made use of Marvin (Cherinka et al. 2017), a core Python package and web framework for MaNGA data, developed by Brian Cherinka, José SánchezGallego, and Brett Andrews. (MaNGA Collaboration, 2017).
This research made use of Astropy, a community-developed core Python package for Astronomy (Astropy Collaboration, 2013).

\section{REFERENCES}

Abolfathi B., et al., 2017, preprint, (arXiv:1707.09322)

Ahn C. P., et al., 2017, ApJ, 839, 72

Becker R. H., White R. L., Helfand D. J., 1995, ApJ, 450, 559

Belfiore F., et al., 2016, MNRAS, 461, 3111

Blanton M. R., et al., 2017, preprint, (arXiv:1703.00052)

Bower R. G., Benson A. J., Malbon R., Helly J. C., Frenk C. S., Baugh C. M., Cole S., Lacey C. G., 2006, MNRAS, 370, 645

Bundy K., et al., 2015, ApJ, 798, 7

Cappellari M., Emsellem E., 2004, PASP, 116, 138

Cherinka B., SÃąnchez-Gallego J., Andrews B., 2017, sdss/marvin: Marvin Beta 2.1.0, doi:10.5281/zenodo.292632, https://doi.org/10.5281/zenodo. 292632

Cheung E., et al., 2016, Nature, 533, 504

Cid Fernandes R., Stasińska G., Mateus A., Vale Asari N., 2011, MNRAS, 413, 1687

Dashyan G., Silk J., Mamon G. A., Dubois Y., Hartwig T., 2017, preprint, (arXiv:1710.05900)

Di Matteo T., Springel V., Hernquist L., 2005, Nature, 433, 604

Drinkwater M. J., Gregg M. D., Hilker M., Bekki K., Couch W. J., Ferguson H. C., Jones J. B., Phillipps S., 2003, Nature, 423,519

Drory N., et al., 2015, AJ, 149, 77

Ferrarese L., Merritt D., 2000, ApJ, 539, L9

Geha M., Blanton M. R., Yan R., Tinker J. L., 2012, ApJ, 757, 85

Gunn J. E., et al., 2006, AJ, 131, 2332

Gürkan G., Hardcastle M. J., Jarvis M. J., 2014, MNRAS, 438,1149

Ilbert O., et al., 2010, ApJ, 709, 644

James P. A., Ivory C. F., 2011, MNRAS, 411, 495

Janz J., Penny S. J., Graham A. W., Forbes D. A., Davies R. L., 2017, preprint, (arXiv:1703.04975)

Jarrett T. H., et al., 2011, ApJ, 735, 112

Kauffmann G., et al., 2003, MNRAS, 346, 1055

Kewley L. J., Heisler C. A., Dopita M. A., Lumsden S., 2001 , ApJS, 132, 37

Ko J., Hwang H. S., Lee J. C., Sohn Y.-J., 2013, ApJ, 767, 90

Krajnović D., Cappellari M., de Zeeuw P. T., Copin Y., 2006, MNRAS, 366, 787

Law D. R., et al., 2015, AJ, 150, 19

Law D. R., et al., 2016, AJ, 152, 83

Lemons S. M., Reines A. E., Plotkin R. M., Gallo E., Greene J. E., 2015, ApJ, 805, 12

Lisker T., Grebel E. K., Binggeli B., 2006, AJ, 132, 497

Moran E. C., Shahinyan K., Sugarman H. R., Vélez D. O., Eracleous M., 2014, AJ, 148, 136

Muñoz R. P., et al., 2015, ApJ, 813, L15

Nucita A. A., Manni L., De Paolis F., Giordano M., Ingrosso G., 2017, ApJ, 837, 66

Peng Y.-j., et al., 2010, ApJ, 721, 193

Penny S. J., Forbes D. A., Pimbblet K. A., Floyd D. J. E., 2014, MNRAS, 443, 3381

Penny S. J., et al., 2016, MNRAS, 462, 3955

Pfeffer J., Baumgardt H., 2013, MNRAS, 433, 1997

Reines A. E., Greene J. E., Geha M., 2013, ApJ, 775, 116

Sartori L. F., Schawinski K., Treister E., Trakhtenbrot B., Koss M., Shirazi M., Oh K., 2015, MNRAS, 454, 3722

Schawinski K., Thomas D., Sarzi M., Maraston C., Kaviraj S., Joo S.-J., Yi S. K., Silk J., 2007, MNRAS, 382, 1415

Schaye J., et al., 2015, MNRAS, 446, 521

Seth A. C., et al., 2014, Nature, 513, 398

Smee S. A., et al., 2013, AJ, 146, 32 
Smethurst R. J., et al., 2016, MNRAS, 463, 2986

Smith R. J., Lucey J. R., Price J., Hudson M. J., Phillipps S., 2012, MNRAS, 419, 3167

Stasińska G., et al., 2008, MNRAS, 391, L29

Toloba E., et al., 2015, ApJ, 799, 172

Wetzel A. R., Tinker J. L., Conroy C., van den Bosch F. C., 2013, MNRAS, 432, 336

Wright E. L., et al., 2010, AJ, 140, 1868

Yan R., et al., 2016a, AJ, 151, 8

Yan R., et al., 2016b, AJ, 152, 197

York D. G., et al., 2000, AJ, 120, 1579

\section{APPENDIX A: STELLAR AND IONISED GAS VELOCITY MAPS}

Stellar and ionised-gas velocity maps are provided in Fig. A1 for the low-mass MaNGA galaxies examined in this work. The ionised gas velocity field is traced using the $\mathrm{H} \alpha$ emission line.

\section{APPENDIX B: BPT DIAGRAMS FOR GALAXIES WITH KINEMATICALLY-OFFSET IONISED GAS}

Spaxel-by-spaxel BPT diagrams are presented in Fig. B1 for the low-mass galaxies in the sample defined in Sec. 2.2 and Sec.3 that host a kinematically-offset ionised gas component. These galaxies typically have AGN-like emission line ratios at their centres.

\section{APPENDIX C: BPT DIAGRAMS FOR GALAXIES WITH CO-ROTATING IONISED \\ GAS}

Spaxel-by-spaxel BPT diagrams are presented in Fig. C1 for the low-mass galaxies in the sample defined in Sec. 2.2 and Sec.3 that host an ionised gas component that is either corotating or counter-rotating with their stellar component. The majority of these galaxies host composite star forming / AGN-like emission line ratios at their centres, and cannot therefore be classed as AGN-host galaxies.

This paper has been typeset from a $\mathrm{T}_{\mathrm{E}} \mathrm{X} / \mathrm{LAT}_{\mathrm{E}} \mathrm{X}$ file prepared by the author. 

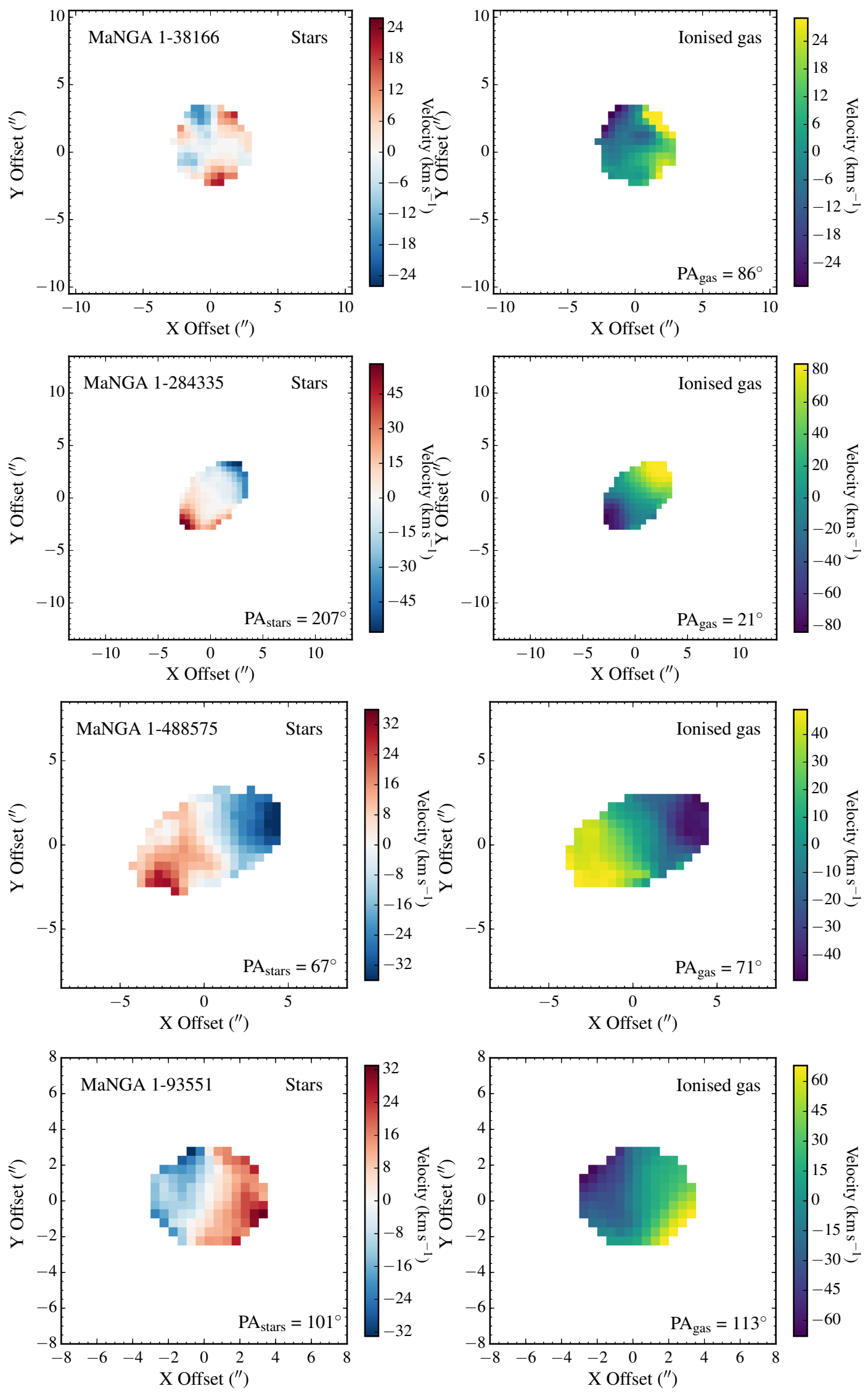

Figure A1. Stellar velocity maps (left panel) and ionised gas velocity maps (right panel) for the low-mass quenched MaNGA galaxies which contain an ionised gas component. The ionised gas velocity fields are traced using the H $\alpha$ line. The maps for the remaining galaxies are shown in Fig.3. 

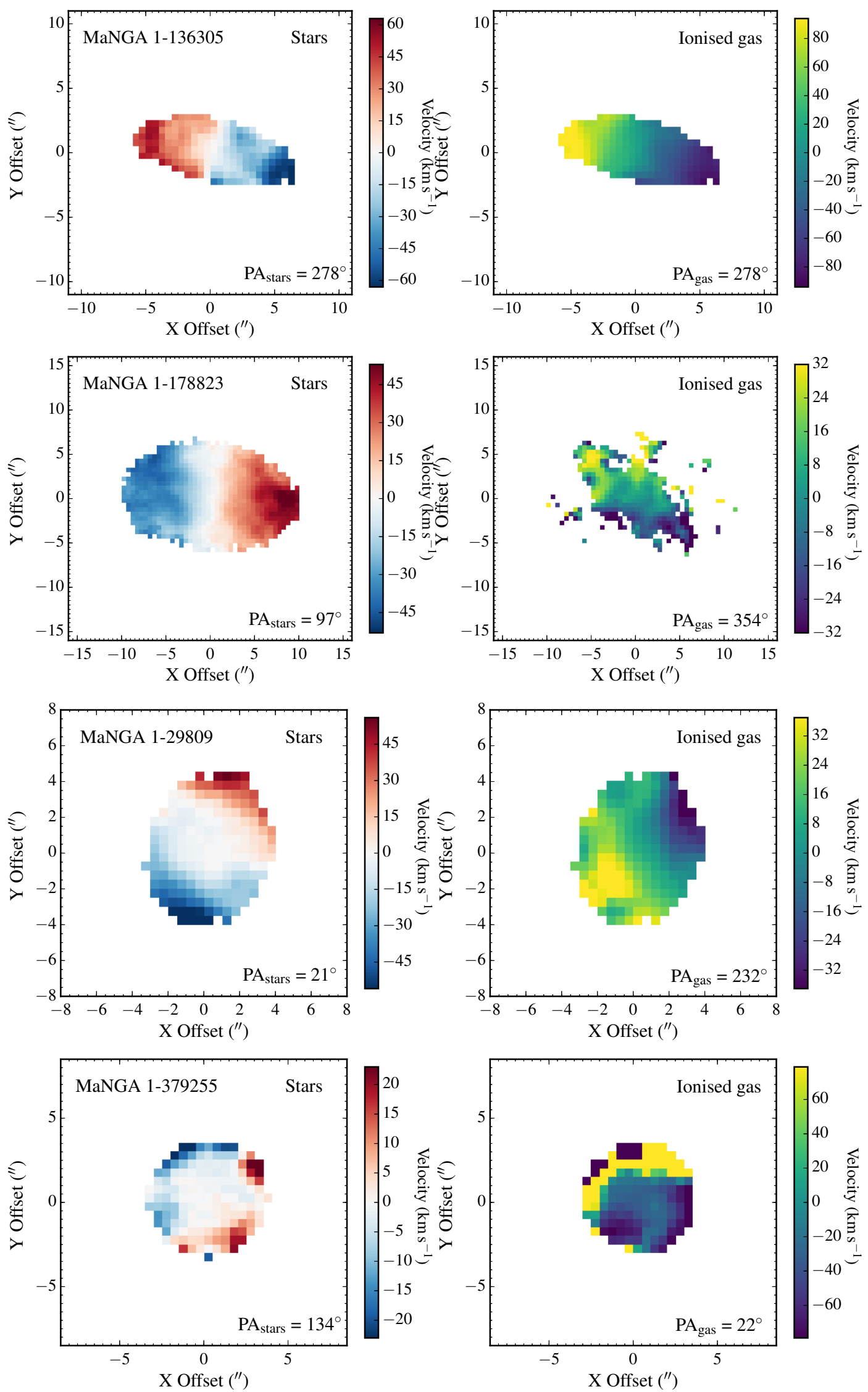

Figure A1 - continued Stellar velocity maps (left panel) and ionised gas velocity maps (right panel) for the low-mass MaNGA galaxies in our sample which contain an ionised gas component. 

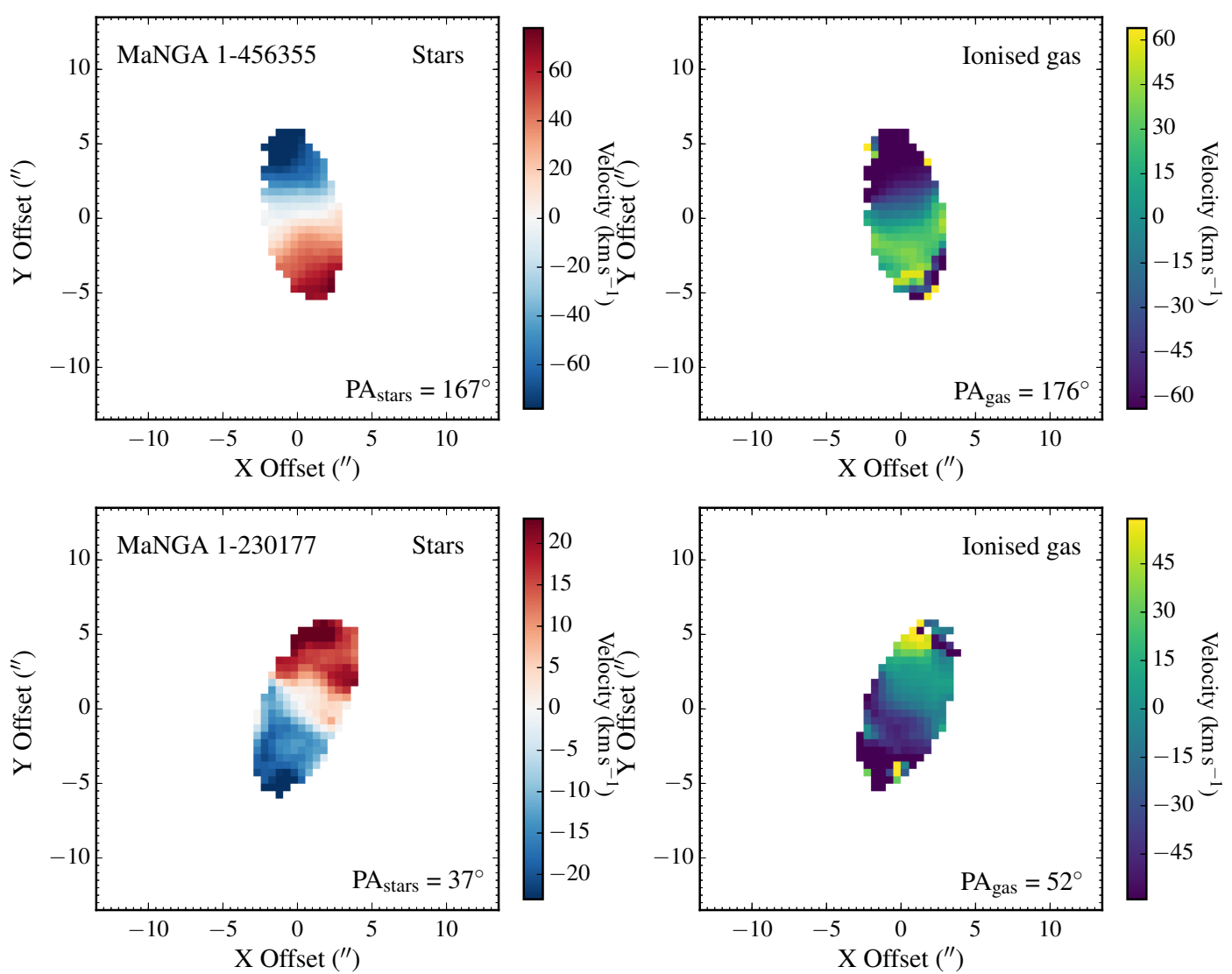

Figure A1 - continued Stellar velocity maps (left panel) and ionised gas velocity maps (right panel) for the low-mass MaNGA galaxies in our sample which contain an ionised gas component. 

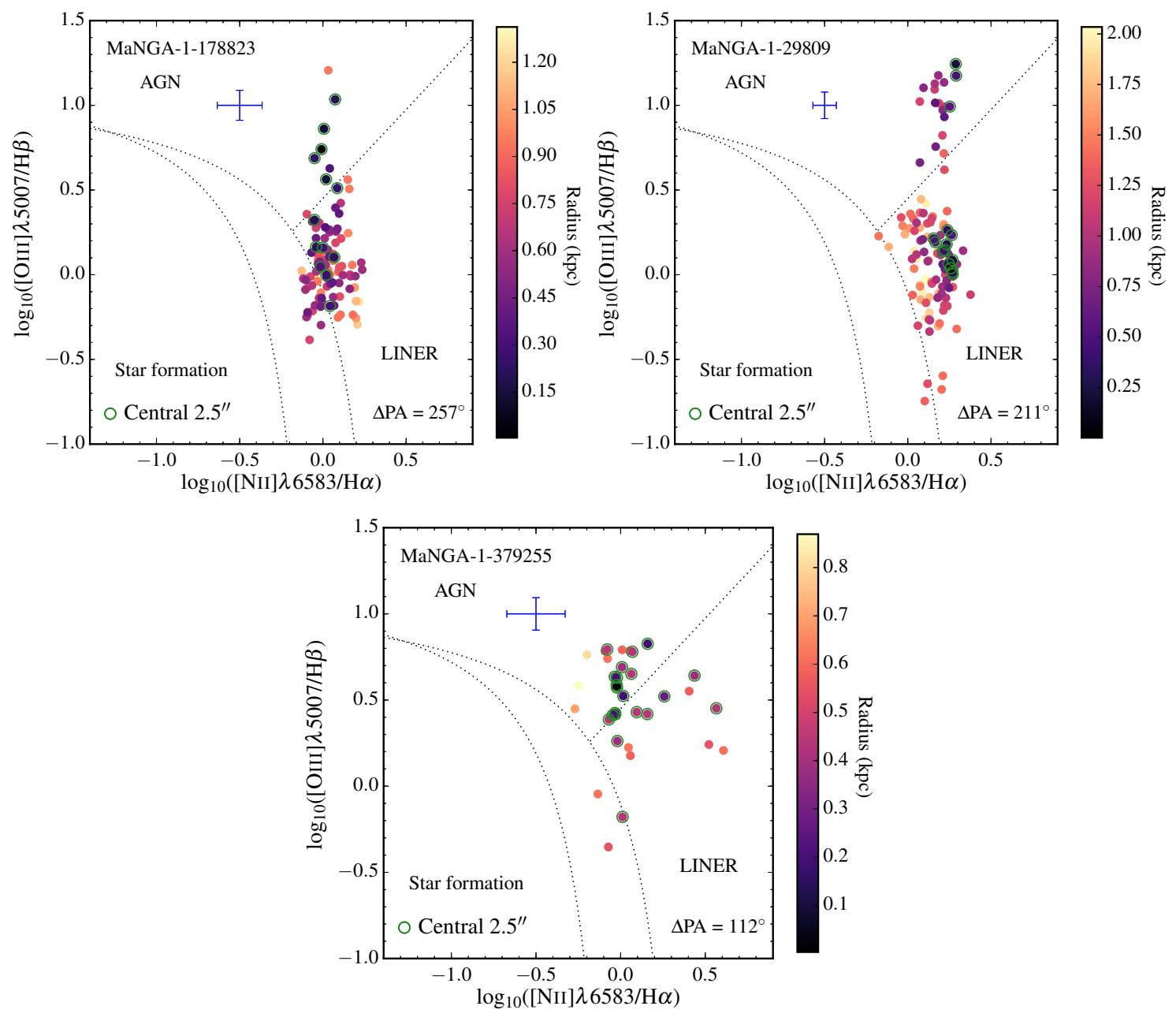

Figure B1. Spaxel-by-spaxel BPT diagrams for the low-mass galaxies with kinematically-offset ionised gas. Spaxels in the central 2.5 arcsecs (the PSF of the reduced datacubes) are circled in green. Also plotted for diagnostic purposes are the Kewley et al. (2001) and Kauffmann et al. (2003) classification lines, which are used to separate extreme starbursts and H II regions from AGN-like emission. The Schawinski et al. (2007) division between Seyfert-like and LINER-like (Low Ionisation Nuclear Emission Region) emission line ratios is also plotted. No object in this sample exhibits central line ratios consistent with star formation. 

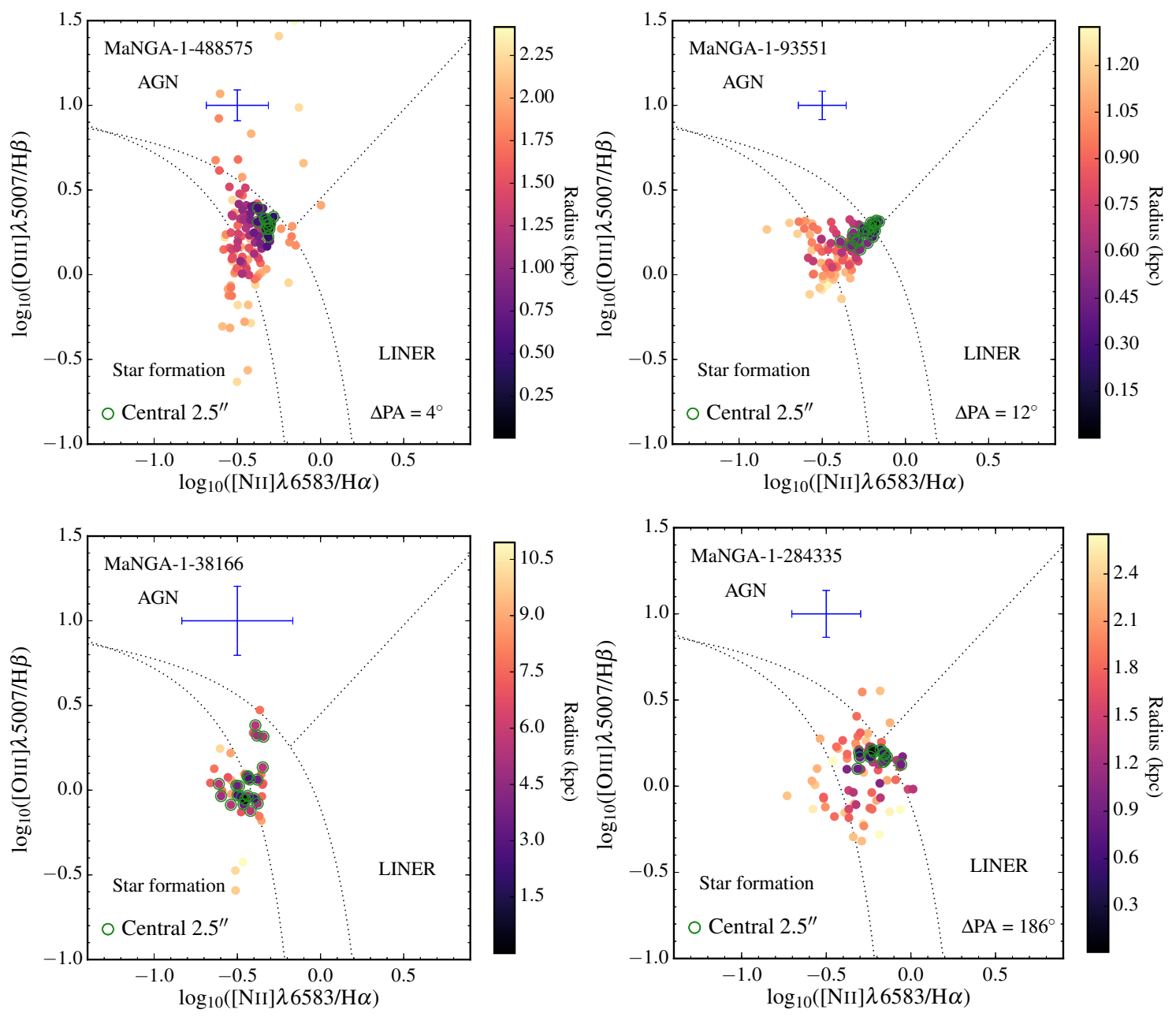

Figure C1. Spaxel-by-spaxel BPT diagrams for the galaxies in our sample with gas that is either co- or counter-rotating from their stellar component. The colour of each point corresponds to its separation from the centre of the galaxy in arcseconds. Spaxels in the central 2.5 arcsecs (the PSF of the reduced datacubes) are circled in green. MaNGA-1-230177 has central spaxels that exhibit emission line ratios consistent with AGN activity. 

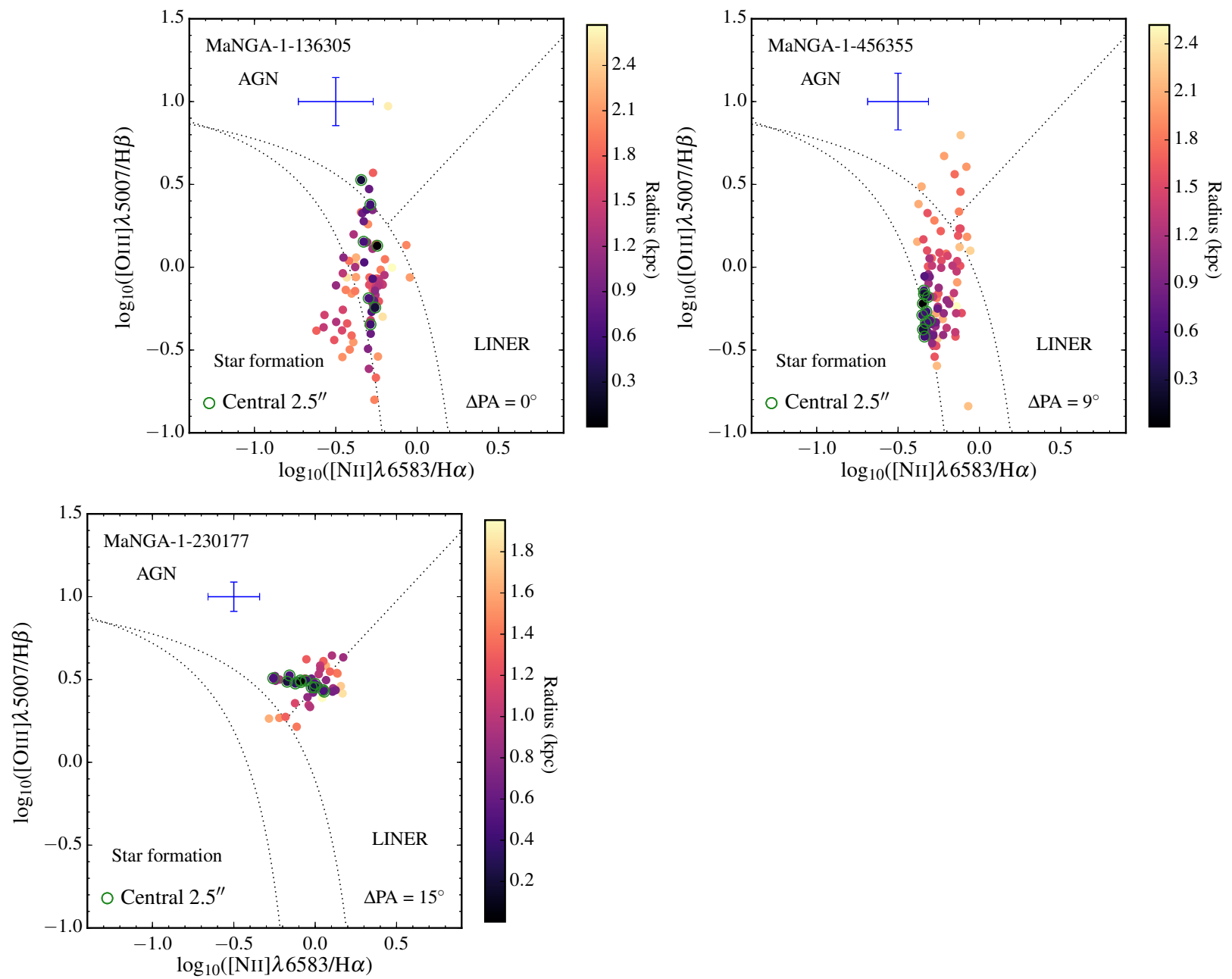

Figure C1 - continued Spaxel-by-spaxel BPT diagrams for low-mass, quenched MaNGA galaxies which host an ionised gas that is either co- or counter-rotating with their stellar component. 